\title{
Reabilitação em pacientes com doença renal crônica em hemodiálise: Uma revisão
}

\section{sistemática de literatura}

\author{
Rehabilitation in chronic kidney disease patients on hemodialysis: A systematic literature review \\ Rehabilitación en pacientes con enfermedad renal crónica en hemodiálisis: Una revisión sistemática
}

\section{de la literatura}

Recebido: 26/01/2022 | Revisado: 03/02/2022 | Aceito: 05/02/2022 | Publicado: 09/02/2022

\author{
Heloisy de Carvalho Cardoso \\ ORCID: https://orcid.org/0000-0001-5590-7521 \\ Centro Universitário Filadélfia, Brasil \\ E-mail: heloisy.carvalho@edu.unifil.br \\ Heloisa Galdino Gumieiro Ribeiro \\ ORCID: https://orcid.org/0000-0002-8175-1740 \\ Centro Universitário Filadélfia, Brasil \\ E-mail: heloisa.ribeiro@unifil.br
}

\begin{abstract}
Resumo
Introdução: A Doença Renal Crônica (DRC) representa uma perda lenta, progressiva e irreversível das funções renais. O tratamento poderá ser desde conservador a terapias substitutivas como a hemodiálise. Em decorrência disto, pacientes submetidos ao tratamento poderão apresentar alterações secundárias a hemodiálise e que poderiam ser tratadas e prevenidas por intervenções fisioterapêuticas através de exercícios físicos aplicados no período intradialítico. Objetivo: Analisar os efeitos da reabilitação fisioterapêutica quando aplicada em pacientes com DRC durante suas sessões de hemodiálise. Métodos: Trata-se de uma revisão sistemática de literatura, que concentrou ensaios clínicos encontrados nas bases de dados: LILACS, PEDro e PubMed, publicados nas línguas portuguesa e inglesa, entre 2016 e 2021, sendo estes posteriormente padronizados conforme Escala PEDro. Resultados: Dos 229 artigos encontrados, 21 ensaios clínicos foram analisados conforme a Escala PEDro. De forma geral foram apresentadas intervenções como exercício aeróbico, resistido, combinado, eletermofototerapia e treinamento muscular respiratório. Com isso, foi possível observar melhora da força muscular, capacidade funcional, capacidade de exercício, da inatividade física, fatores de risco cardiovasculares e melhora da qualidade do sono, além de proporcionar melhora de aspectos relacionados aos biomarcadores inflamatórios e qualidade da diálise. Tais exercícios foram realizados com uma média de 30 a 40 minutos, três vezes na semana, por uma média de 12 semanas. Conclusão: Pode-se concluir que um programa de reabilitação intradialítica pode ofertar efeitos positivos ao paciente em hemodiálise. Porém, sugere-se que a qualidade de vida destes pacientes seja tratada como desfecho primário, obtendo-se assim uma reabilitação de pacientes com DRC amplamente atendida.
\end{abstract}

Palavras-chave: Reabilitação; Insuficiência renal crônica; Diálise renal; Fisioterapia.

\begin{abstract}
Introduction: Chronic Kidney Disease (CKD) represents a slow, progressive and irreversible loss of kidney function. Treatment may range from conservative to substitutive therapies such as hemodialysis. As a result, patients undergoing treatment may present secondary changes to hemodialysis that could be treated and prevented by physical therapy interventions through physical exercises applied in the intradialytic period. Objective: To analyze the effects of physiotherapeutic rehabilitation when applied in patients with CKD during hemodialysis sessions. Methods: This is a systematic literature review, which concentrated clinical trials found in the databases: LILACS, PEDro and PubMed, published in Portuguese and English languages, between 2016 and 2021, which were subsequently standardized according to the PEDro Scale. Results: Of the 229 articles found, 21 clinical trials were analyzed. In general, interventions such as aerobic exercise, resistance exercise, combined exercise, thermotherapy and respiratory muscle training were presented. Furthermore, it was possible to observe improvement in muscle strength, functional capacity, exercise capacity, physical inactivity, cardiovascular risk factors, and improvement in sleep quality, besides providing improvement in aspects related to inflammatory biomarkers and quality of dialysis. These exercises were performed with an average of 30 to 40 minutes, three times a week, for an average of 12 weeks. Conclusion: It can be concluded that an intradialytic rehabilitation program can offer positive effects to the patient on hemodialysis. However it is suggested that the quality of life of these patients should be treated as a primary outcome, getting this way a complete and wide rehabilitation of patients with CKD.
\end{abstract}

Keywords: Rehabilitation; Chronic kidney insufficiency; Renal dialysis; Physiotherapy. 


\section{Resumen}

Introducción: La enfermedad renal crónica (ERC) representa una pérdida lenta, progresiva e irreversible de la función renal. El tratamiento puede ir desde terapias conservadoras hasta terapias de sustitución, como la hemodiálisis. Los pacientes en tratamiento pueden presentar alteraciones secundarias a la hemodiálisis que podrían ser tratadas y prevenidas mediante intervenciones fisioterapéuticas aplicadas en periodo intradiálisis. Objetivo: Analizar los efectos de la rehabilitación fisioterapéutica cuando se aplica a pacientes con ERC durante sus sesiones de hemodiálisis. Métodos: Se trata de una revisión sistemática de la literatura, que concentró los ensayos clínicos encontrados en bases de datos: LILACS, PEDro y PubMed, publicados en portugués e inglés, entre 2016 y 2021, que fueron estandarizados según la Escala PEDro. Resultados: De los 229 artículos encontrados, se analizaron 21 ensayos clínicos. En general, se presentaron intervenciones como ejercicios aeróbicos, ejercicios resistidos, ejercicios combinados, termoterapia y entrenamiento de los músculos respiratorios. Estos mostraron una mejora en la fuerza muscular, capacidad funcional, capacidad de ejercicio, inactividad física, factores de riesgo cardiovascular y mejora en la calidad del sueño, además de proporcionar una mejora en los biomarcadores inflamatorios y la calidad de la diálisis. Estos ejercicios se realizaron durante una media de 30-40 minutos, tres veces por semana, durante una media de 12 semanas. Conclusión: Se puede concluir que un programa de rehabilitación intradiálisis puede ofrecer efectos positivos para el paciente de hemodiálisis. Sin embargo, sugiere que la calidad de vida sea tratada como resultado primario, obteniendo así una rehabilitación de los pacientes con ERC de forma integral.

Palabras clave: Rehabilitación; Insuficiencia renal crónica; Diálisis renal; Fisioterapia.

\section{Introdução}

Com o passar dos anos e com o aumento progressivo e significativo dos índices de doenças crônicas e degenerativas, a doença renal tem ganhado maior visibilidade e muitos estudos da área da saúde têm sido voltados para ela. Segundo o Ministério da Saúde (2014) atualmente as doenças renais crônicas já podem ser consideradas como um problema de saúde pública por suas elevadas taxas de morbimortalidade (Brasil, 2014).

Portanto, segundo a Sociedade Brasileira de Nefrologia (SBN), a Doença Renal Crônica (DRC) representa uma perda lenta, progressiva e irreversível das funções renais, gerando assim ao organismo a incapacidade de manter seu equilíbrio metabólico e hidroeletrolítico, que depende diretamente da filtração glomerular que estará prejudicada.

As funções renais podem ser aferidas através de um simples exame de urina e também por exames mais detalhados, caso seja necessário, dependendo do paciente e seu caso. Porém, é a Taxa de Filtração Glomerular (TFG) que poderá trazer indicativos reais de declínio da função renal, e atualmente é considerado segundo o Ministério da Saúde portador de DRC qualquer indivíduo que, independente da causa, apresente por pelo menos três meses consecutivos uma TFG $<60 \mathrm{ml} / \mathrm{min} / 1,73 \mathrm{~m}^{2}$ (Sociedade Brasileira de Nefrologia [SBN], 2021; Brasil, 2014).

Sendo assim, com base na classificação da DRC, o paciente irá receber adequadamente o tratamento voltado ao seu quadro clínico e suas reais necessidades. A hemodiálise, consiste em um tipo de terapia substitutiva no qual uma máquina filtra o sangue do paciente, de forma extracorpórea, coletando-o através de um acesso vascular, que pode ser um cateter central ou uma fístula arteriovenosa. Em seguida o sangue é impulsionado por uma bomba até o filtro de diálise (dialisador), no qual é exposto à uma solução de diálise através de uma membrana que retira o líquido e as toxinas em excesso. O sangue é devolvido para o paciente pelo mesmo acesso (SBN, 2021).

O processo de tratamento por hemodiálise pode levar de 3 a 5 horas, aplicado de 2 a 5 dias da semana, dependendo do estágio da doença, chegando em casos mais graves a serem realizados diariamente. Com uma terapêutica voltada para o tratamento contínuo, a expectativa de vida destes pacientes aumentou nos últimos anos, porém outros fatores podem estar envolvidos para que qualidade de vida ainda se mantenha em queda (Lima et al., 2021).

Diversas complicações dos demais sistemas que compõem o corpo humano podem surgir com a hemodiálise, sendo possivelmente permanentes devido ao tratamento de longa duração. Estes pacientes podem vir a apresentar alterações físicas importantes pelas restrições impostas pela doença e pelo tratamento, alterações estas que acarretam em inúmeras complicações à longo prazo (Ramos et al., 2014). 
Portanto, a aplicação de programas de reabilitação fisioterapêutica pode contribuir positivamente quando também aplicado durante o período intradialítico destes pacientes com o objetivo de sanar complicações do tratamento em si, além do papel preventivo, onde se busca minimizar as complicações secundárias, que influencia diretamente na melhora de sua qualidade de vida e na realização de suas atividades de vida diária (AVD’s). Desta forma, o objetivo deste trabalho é analisar os efeitos da reabilitação fisioterapêutica quando aplicada em pacientes com DRC durante suas sessões de hemodiálise, além de classificar as intervenções realizadas e suas características.

\section{Metodologia}

A presente pesquisa trata-se de uma revisão sistemática de literatura, realizada de acordo com o PRISMA, que concentrou e analisou os resultados obtidos através de programas de reabilitação aplicados em pacientes com DRC durante a realização de hemodiálise (Prisma, 2020).

A busca dos artigos foi realizada por um avaliador único, concentrando estudos entre as datas de 19 de junho a 22 de agosto nos bancos de dados eletrônicos LILACS, PEDro e PubMed através dos seguintes descritores: hemodiálise (hemodialysis), fisioterapia (physiotherapy), reabilitação (rehabilitation).

Foram selecionados ensaios clínicos publicados em língua portuguesa e inglesa, com data de publicação entre 2016 e 2021, sendo incluídos aqueles que se tratavam acerca de um programa de reabilitação voltado para pacientes com DRC, realizado durante suas sessões de hemodiálise, não distinguidos entre gêneros ou idade, ou ainda, tempo de início de tratamento substitutivo de hemodiálise. Todos os artigos foram analisados conforme a padronização da Escala PEDro, e inseridos apenas aqueles com pontuação igual ou superior à 6 .

Foram excluídos aqueles artigos que não realizaram um programa de reabilitação voltado ao paciente renal, e principalmente o qual não seja aplicado durante a sessão de hemodiálise, excluindo, portanto, aqueles realizados anteriormente, posteriormente ou que realizassem exercícios domiciliares.

Os artigos foram selecionados pela simples leitura do título, sendo descartados aqueles evidentemente não relacionados ao objetivo do trabalho. Para os potencialmente elegíveis, foram avaliados os resumos, sendo selecionados aqueles que se adequaram aos critérios de inclusão pré-definidos. Dessa maneira, os artigos que apresentarem todos os critérios de inclusão foram analisados na íntegra e por fim, foram sistematizados seguindo a padronização da escala PEDro e apresentados através de tabelas (Escala PEDro - PEDro, 1999).

A Escala PEDro se trata de um método avaliativo, a fim de auxiliar os usuários e pesquisadores quanto à qualidade metodológica de ensaios clínicos aleatorizados, sendo baseada na Escala Delphi. Apresenta-se através de 11 critérios avaliativos, sendo sua pontuação máxima um escore de 10 pontos (Shiwa et al., 2011).

São incluídos na escala critérios de elegibilidade dos estudos (critério 1), este não pontuado. Mas também informações quanto à alocação, homogeneidade da amostra, cegamento de pacientes, terapeutas e avaliadores. E ainda, aspectos relacionados aos desfechos encontrados, de acordo com as mensurações realizadas, realização de controle ou intervenção para pelo menos um resultado-chave investigado e ainda, comparações estatísticas e medidas de precisão dos estudos (Shiwa et al., 2011).

Após avaliação de todos os critérios estipulados na escala, os resultados foram sintetizados em uma tabela, apresentando dados relacionados a duração em semanas das intervenções e a frequência semanal das intervenções aplicadas. E ainda, as modalidades de intervenções, os materiais utilizados para aplicá-las e o tempo de intervenção em minutos. Com isso, apresentou-se também os desfechos encontrados em cada um dos estudos. 


\section{Resultados}

Foram selecionados para a análise final 21 artigos, sendo dois publicados no ano de 2016, três em 2017, oito no ano de 2018, quatro em 2019 e quatro no ano de 2020. Na Figura 1 pode ser observado o processo de seleção.

A Tabela 1 traz a classificação dos artigos conforme a Escala PEDro dos artigos que indispensavelmente atingiram todos os objetivos gerais e específicos do presente estudo. Todas as suas características de pontuação podem ser observadas nesta tabela. Já na Tabela 2 são apresentadas as características dos 21 ensaios clínicos que aplicaram diferentes formas de intervenções exclusivamente intradialíticas. A caracterização geral dos estudos analisados está descrita na tabela 3

Os estudos incluíram em geral, pacientes com uma média de 54.7 anos $( \pm 9.25)$, que realizavam hemodiálise por no mínimo seis meses, sem qualquer distinção entre sexos, feminino ou masculino, e ainda, não foram descritos os tipos de acessos vasculares utilizados pelos pacientes, durantes as intervenções.

Os artigos em questão trazem como principais intervenções intradialíticas a aplicação de exercícios resistidos, aeróbicos ou combinados, que representa a associação dos exercícios resistidos e aeróbicos numa única sessão. Além destes, foram citadas também a eletroestimulação neuromuscular, terapia por fotobiomodulação, o treinamento muscular respiratório e a compressão pneumática como formas de reabilitação aplicadas durante as sessões de hemodiálise.

Quanto à frequência da reabilitação intradialítica, dois estudos foram realizados em 24 semanas $(9,6 \%)$, sete em 12 semanas $(33,3 \%)$, um em 16 semanas $(4,8 \%)$, seis em 8 semanas $(28,5 \%)$, três em 4 semanas $(14,2 \%)$ e dois em 3 semanas $(9,6 \%)$. Em suma, 85,7\% aplicaram seus protocolos de reabilitação três vezes por semana, 9,5\% realizados duas vezes por semana e apenas $4,8 \%$ dos estudos foram realizados apenas uma vez por semana.

Figura 1 - Fluxograma do processo de seleção dos artigos, segundo base de dados, de acordo com o PRISMA.

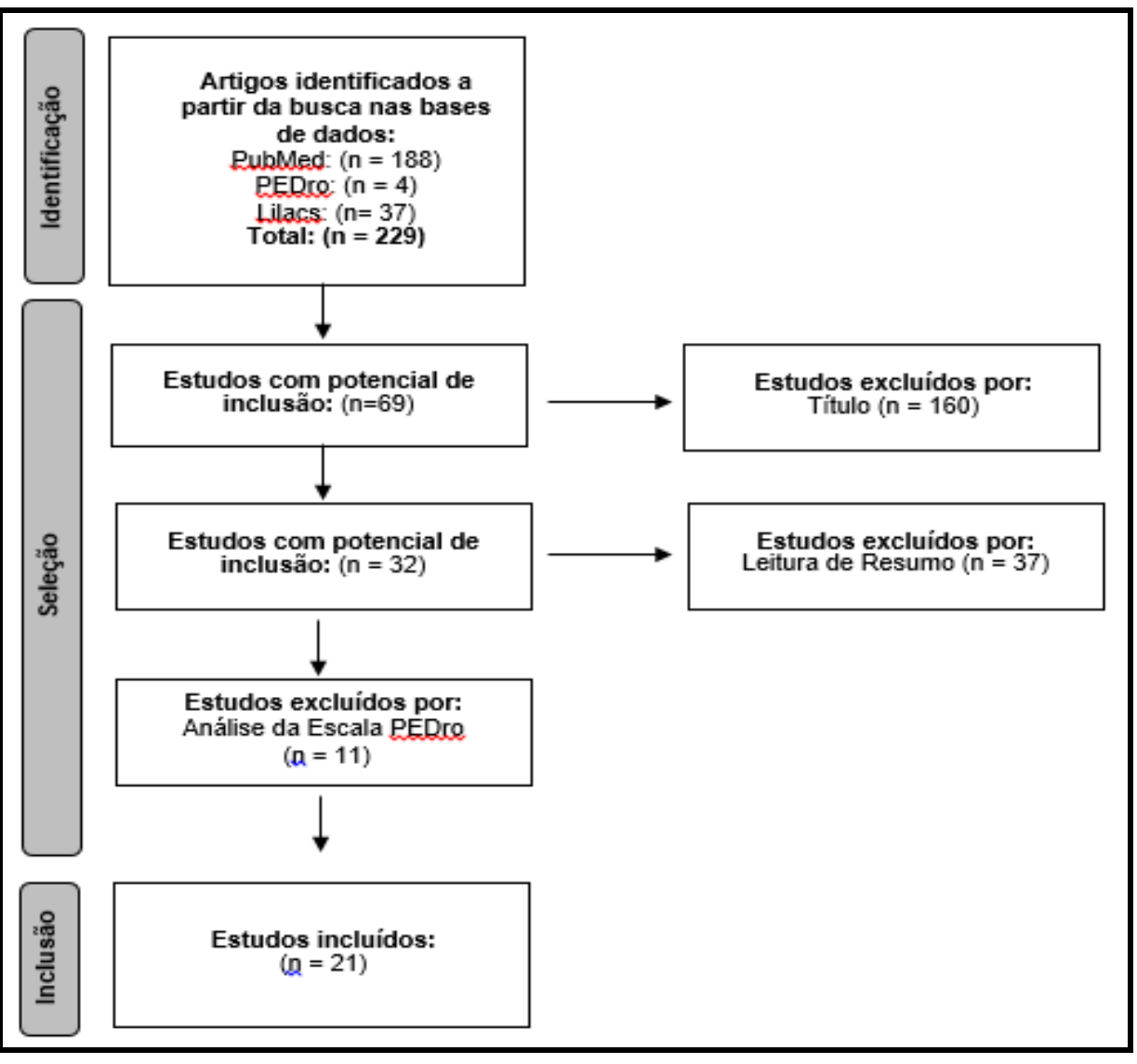

Fonte: Figura desenvolvida pelas autoras. 
Tabela 1 - Apresentação dos artigos analisados e pontuação conforme Escala PEDro.

\begin{tabular}{|c|c|c|c|c|c|c|c|c|c|c|c|c|}
\hline \multirow[b]{2}{*}{ ARTIGOS } & \multicolumn{12}{|c|}{ CRITÉRIOS } \\
\hline & 1 & 2 & 3 & 4 & 5 & 6 & 7 & 8 & 9 & 10 & 11 & $\begin{array}{l}\text { TOTAL } \\
\text { (pontos) }\end{array}$ \\
\hline $\begin{array}{l}\text { Effect of resistance exercises on the indicators of muscle reserves and handgrip strength in adult } \\
\text { patients on hemodialysis }\end{array}$ & $\mathrm{x}$ & $\mathrm{x}$ & $\mathrm{x}$ & $\mathrm{x}$ & $\mathrm{x}$ & $\mathrm{x}$ & $\mathrm{x}$ & $\mathrm{x}$ & $\mathrm{x}$ & $\mathrm{x}$ & $\mathrm{x}$ & 10 \\
\hline $\begin{array}{l}\text { Impact of neuromuscular electrical stimulation on functional capacity of patients with chronic } \\
\text { kidney disease on hemodialysis }\end{array}$ & $\mathrm{x}$ & $\mathrm{x}$ & $\mathrm{x}$ & $\mathrm{x}$ & & & & $\mathrm{x}$ & $\mathrm{x}$ & $\mathrm{x}$ & $\mathrm{x}$ & 7 \\
\hline $\begin{array}{l}\text { Effects of neuromuscular electrical stimulation during hemodialysis on peripheral muscle strength } \\
\text { and exercise capacity: a randomized clinical trial }\end{array}$ & $\mathrm{x}$ & $\mathrm{x}$ & $\mathrm{x}$ & $\mathrm{x}$ & $\mathrm{x}$ & & $\mathrm{x}$ & $\mathrm{x}$ & $\mathrm{x}$ & $\mathrm{x}$ & $\mathrm{x}$ & 9 \\
\hline Pneumatic compression, but not exercise, can avoid intradialytic hypotension: a randomized trial & $\mathrm{x}$ & $\mathrm{x}$ & $\mathrm{x}$ & $\mathrm{x}$ & & & & $\mathrm{x}$ & $\mathrm{x}$ & $\mathrm{x}$ & $\mathrm{x}$ & 7 \\
\hline $\begin{array}{l}\text { Effects of intradialytic neuromuscular electrical stimulation on strength and muscle architecture in } \\
\text { patients with chronic kidney failure: randomized clinical trial }\end{array}$ & $\mathrm{x}$ & $\mathrm{x}$ & $\mathrm{x}$ & $\mathrm{x}$ & & & & & $\mathrm{x}$ & $\mathrm{x}$ & $\mathrm{x}$ & 6 \\
\hline $\begin{array}{l}\text { Effect of intradialytic exercise on daily physical activity and sleep quality in maintenance } \\
\text { hemodialysis patients }\end{array}$ & $\mathrm{x}$ & $\mathrm{x}$ & $\mathrm{x}$ & $\mathrm{x}$ & $\Gamma$ & & & & $\mathrm{x}$ & $\mathrm{x}$ & $\mathrm{x}$ & 6 \\
\hline $\begin{array}{l}\text { Effect of continuous progressive resistance training during hemodialysis on body composition, } \\
\text { physical function and quality of life in end-stage renal disease patients: a randomized controlled } \\
\text { trial. }\end{array}$ & $\mathrm{x}$ & $\mathrm{x}$ & $\mathrm{x}$ & $\mathrm{x}$ & & & & & $\mathrm{x}$ & $\mathrm{x}$ & $\mathrm{x}$ & 6 \\
\hline $\begin{array}{l}\text { Effects of the inspiratory muscle training and aerobic training on respiratory and functional } \\
\text { parameters, inflammatory biomarkers, redox status and quality of life in hemodialysis patients: a } \\
\text { randomized clinical trial. }\end{array}$ & $\mathrm{x}$ & $\mathrm{x}$ & $\mathrm{x}$ & $\mathrm{x}$ & & & $\mathrm{x}$ & $\mathrm{x}$ & $\mathrm{x}$ & $\mathrm{x}$ & $\mathrm{x}$ & 8 \\
\hline $\begin{array}{l}\text { Acute effect of photobiomodulation therapy on handgrip strength of chronic kidney disease patients } \\
\text { during hemodialysis. }\end{array}$ & $\mathrm{x}$ & $\mathrm{x}$ & & $\mathrm{x}$ & $\mathrm{x}$ & $\mathrm{x}$ & & & $\mathrm{x}$ & $\mathrm{x}$ & $\mathrm{x}$ & 7 \\
\hline $\begin{array}{l}\text { Beneficial effect of intradialytic electrical muscle stimulation in hemodialysis patients: a } \\
\text { randomized controlled trial. }\end{array}$ & $\mathrm{x}$ & $\mathrm{x}$ & $\mathrm{x}$ & $\mathrm{x}$ & & & $\mathrm{x}$ & $\mathrm{X}$ & $\mathrm{x}$ & $\mathrm{x}$ & $\mathrm{x}$ & 8 \\
\hline The effect of transcutaneous electrical nerve stimulation (TENS) on increasing salivary & $\mathrm{x}$ & $\mathrm{x}$ & $\mathrm{x}$ & $\mathrm{x}$ & $\mathrm{x}$ & & & $\mathrm{x}$ & $\mathrm{x}$ & $\mathrm{x}$ & $\mathrm{x}$ & 8 \\
\hline
\end{tabular}


hemodialysis patients.

\begin{tabular}{|c|c|c|c|c|c|c|c|c|c|c|c|c|}
\hline $\begin{array}{l}\text { Virtual Reality Exercise Intradialysis to Improve Physical Function: A Feasibility Randomized } \\
\text { Trial. }\end{array}$ & $\mathrm{x}$ & $\mathrm{x}$ & $\mathrm{x}$ & $x$ & & | & 1 & $\mathrm{x}$ & $\mathrm{x}$ & $\mathrm{x}$ & $\mathrm{x}$ & 7 \\
\hline Influence of Physical Exercise on the Dialytic Adequacy Parameters of Patients on Haemodialysis. & $\mathrm{x}$ & $\mathrm{x}$ & $\mathrm{x}$ & $\mathrm{x}$ & & $T$ & T & & $\mathrm{x}$ & $\mathrm{x}$ & $\mathrm{x}$ & 6 \\
\hline $\begin{array}{l}\text { Effects of intradialytic resistance exercise on systemic inflammation in maintenance hemodialysis } \\
\text { patients with sarcopenia: a randomized controlled trial }\end{array}$ & $\mathrm{x}$ & $\mathrm{x}$ & & $\mathrm{x}$ & & & $T$ & $\mathrm{x}$ & $\mathrm{x}$ & $\mathrm{x}$ & $\mathrm{x}$ & 6 \\
\hline $\begin{array}{l}\text { Aerobic exercise training and nontraditional cardiovascular risk factors in hemodialysis patients: } \\
\text { results from a prospective randomized trial. }\end{array}$ & $\mathrm{x}$ & $\mathrm{x}$ & & $x$ & & & $\mathrm{x}$ & $\mathrm{x}$ & $\mathrm{X}$ & $\mathrm{x}$ & $\mathrm{x}$ & 7 \\
\hline $\begin{array}{l}\text { The effect of intradialytic exercise twice a week on the physical capacity, inflammation, and } \\
\text { nutritional status of dialysis patients: a randomized controlled trial. }\end{array}$ & $x$ & $\mathrm{X}$ & & $\mathrm{x}$ & & $T$ & $T$ & $\mathrm{x}$ & $\mathrm{X}$ & $\mathrm{x}$ & $\mathrm{X}$ & 6 \\
\hline $\begin{array}{l}\text { The effect of intradialytic combined exercise on hemodialysis efficiency in end-stage renal disease } \\
\text { patients: a randomized-controlled trial. }\end{array}$ & $\mathrm{x}$ & $\mathrm{X}$ & & $\mathrm{x}$ & & & $\mathrm{x}$ & & $\mathrm{X}$ & $\mathrm{x}$ & $\mathrm{x}$ & 6 \\
\hline $\begin{array}{l}\text { Photobiomodulation therapy increases functional capacity of patients with chronic kidney failure: } \\
\text { randomized controlled trial. }\end{array}$ & $\mathrm{x}$ & $\mathrm{X}$ & $\mathrm{X}$ & $x$ & & $\mathrm{x}$ & $x$ & $\mathrm{X}$ & $\mathrm{X}$ & $\mathrm{x}$ & $\mathrm{x}$ & 9 \\
\hline $\begin{array}{l}\text { Twelve-week intradialytic cycling exercise improves physical functional performance with gain in } \\
\text { muscle strength and endurance: a randomized controlled trial. }\end{array}$ & $\mathrm{x}$ & $\mathrm{X}$ & $\mathrm{x}$ & $\mathrm{x}$ & & & & & $\mathrm{X}$ & $\mathrm{x}$ & $\mathrm{x}$ & 6 \\
\hline $\begin{array}{l}\text { Effectiveness of inspiratory muscle training on respiratory fitness and breathlessness in chronic } \\
\text { renal failure: A randomized control trial. }\end{array}$ & $\mathrm{x}$ & $\mathrm{X}$ & & $\mathrm{x}$ & $\mathrm{x}$ & & $\mathrm{x}$ & $\mathrm{X}$ & $\mathrm{X}$ & $\mathrm{x}$ & $\mathrm{x}$ & 8 \\
\hline $\begin{array}{l}\text { Exercise for people living with frailty and receiving haemodialysis: a mixed methods randomised } \\
\text { controlled feasibility study. }\end{array}$ & $\mathrm{x}$ & $\mathrm{X}$ & & $\mathrm{x}$ & & & $T$ & $\mathrm{x}$ & $\mathrm{X}$ & $\mathrm{x}$ & $\mathrm{x}$ & 6 \\
\hline
\end{tabular}

Fonte: Tabela desenvolvida pelos autores. 


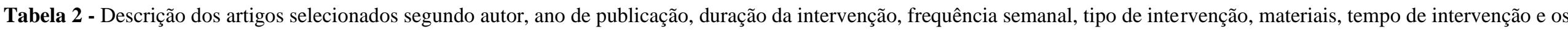
principais desfechos encontrados.

\begin{tabular}{|c|c|c|c|c|c|c|}
\hline Estudo & $\begin{array}{l}\text { Duração } \\
\text { (semanas) }\end{array}$ & $\begin{array}{l}\text { Frequência } \\
\text { semanal }\end{array}$ & Intervenção & Materiais & $\begin{array}{l}\text { Tempo de } \\
\text { Intervenção }\end{array}$ & Desfechos \\
\hline Soto et al. (2016) & $\begin{array}{l}12 \text { semanas }+ \\
\text { Follow-up entre } \\
\text { Dezembro de } \\
2013 \text { e Junho de } \\
2012 .\end{array}$ & $2 \mathrm{x}$ semana. & Exercícios resistidos. & Caneleira e Theraband. & 50 minutos. & $\begin{array}{l}\uparrow \text { da circunferência muscular do braço, da área muscular do braço } \\
\text {, e da força de punho. }\end{array}$ \\
\hline$\underset{(2016)}{\mathrm{ROXO}} \mathrm{AL}$ & 8 semanas. & 3x semana. & $\begin{array}{l}\text { Estimulação elétrica } \\
\text { neuromuscular em } \\
\text { quadríceps femoral. }\end{array}$ & $\begin{array}{l}\text { Neurodyn II } \\
\text { (Ibramed), estimulador } \\
\text { elétrico neuromuscular. }\end{array}$ & 30 minutos. & $\begin{array}{c}\uparrow \text { da pressão inspiratória máxima, do teste de } 1 \mathrm{RM} \text { e a distância } \\
\text { percorrida no TC6min. } \downarrow \text { da pressão arterial sistólica e frequência } \\
\text { respiratória. }\end{array}$ \\
\hline $\begin{array}{l}\text { BRUGGERMANN } \\
\text { ET AL. (2017) }\end{array}$ & 4 semanas. & $3 \mathrm{x}$ semana. & $\begin{array}{c}\text { Estimulação elétrica } \\
\text { neuromuscular de alta } \\
\text { frequência e intensidade } \\
\text { (HG), vs. baixa frequência e } \\
\text { intensidade (LG), em } \\
\text { quadríceps. }\end{array}$ & $\begin{array}{l}\text { Estimulador muscular } \\
\text { portátil de canal duplo } \\
\text { (Carci Fesmed IV) e } \\
\text { eletrodos auto-adesivos. }\end{array}$ & 60 minutos. & $\begin{array}{c}\uparrow \text { na FM periférica em altas frequências e intensidades (HG). E } \uparrow \\
\text { da distância do TC6min em ambos os grupos. Apenas LG } \uparrow \text { os } \\
\text { níveis de IGF-1 e, apenas HG } \downarrow \text { os níveis de IL-10. }\end{array}$ \\
\hline $\begin{array}{l}\text { ÁLVARES ET AL. } \\
(2017)\end{array}$ & 3 semanas. & $3 x$ semana & $\begin{array}{l}\text { Exercício aeróbico de } \\
\text { ciclismo vs. compressão } \\
\text { pneumática. }\end{array}$ & $\begin{array}{c}\text { Cicloergômetro } \\
\text { (Monark), Sistema de } \\
\text { Compressão Pneumática } \\
\text { ( Kendall 7,325- } \\
\text { Covidien). }\end{array}$ & 60 minutos. & $\begin{array}{c}\text { A compressão pneumática } \downarrow \text { a hipotensão induzida por } \\
\text { Hemodiálise, não houveram efeitos nos fluidos. Nem o exercício } \\
\text { e nem a compressão pneumática aumentaram a remoção de } \\
\text { fosfato. }\end{array}$ \\
\hline $\begin{array}{l}\text { SCHARDONG ET } \\
\text { AL. (2017) }\end{array}$ & 8 semanas. & $3 x$ semana. & $\begin{array}{l}\text { Estimulação elétrica } \\
\text { neuromuscular em } \\
\text { quadríceps. }\end{array}$ & $\begin{array}{c}\text { Estimulador elétrico } \\
\text { calibrado (Neurodyn II, } \\
\text { modelo N53) e eletrodos } \\
\text { autocolantes (ValuTrode, } \\
\text { modelo CF7515). }\end{array}$ & $\begin{array}{l}20-34 \text { minutos, } \\
\text { com } \\
\text { incremento de } \\
2 \text { minutos por } \\
\text { semana. }\end{array}$ & $\begin{array}{l}\uparrow \text { na força dos membros inferiores no GI e na proteção da atrofia } \\
\text { muscular, porém, não houve mudança na capacidade funcional e } \\
\text { na função endotelial para ambos os grupos. }\end{array}$ \\
\hline CHO ET AL. (2018) & 12 semanas. & $3 x$ semana. & $\begin{array}{l}\text { Exercício aeróbico, vs. } \\
\text { exercícios resistidos vs. } \\
\text { exercícios combinados. }\end{array}$ & $\begin{array}{c}\text { Bicicleta ergométrica e } \\
\text { TheraBand. }\end{array}$ & 40 minutos. & $\begin{array}{c}\uparrow \text { do equivalente metabólico, maior no grupo de exercícios } \\
\text { combinados. } \downarrow \text { do número total de sedentários no grupo de } \\
\text { exercício aeróbico. E, } \downarrow \text { do índice médio de fragmentação do } \\
\text { sono. }\end{array}$ \\
\hline
\end{tabular}




\begin{tabular}{|c|c|c|c|c|c|c|}
\hline$\underset{(2018)}{\text { ROSA }}$ & 12 semanas. & $3 x$ semana. & $\begin{array}{l}\text { Treinamento de resistência } \\
\text { progressiva. }\end{array}$ & $\begin{array}{l}\text { Pesos livres e } \\
\text { Therabands. }\end{array}$ & 40-50 minutos. & $\begin{array}{c}\uparrow \text { Massa magra da perna, tamanho do efeito conteúdo mineral } \\
\text { ósseo, força da perna em repetições e flexibilidade. Capacidade } \\
\text { de caminhada, força muscular periférica e qualidade de vida não } \\
\text { foram } \\
\text { diferentes entre os grupos. }\end{array}$ \\
\hline $\begin{array}{l}\text { FIGUEIREDO ET } \\
\text { AL. (2018) }\end{array}$ & $\begin{array}{l}8 \text { semanas }+ \\
\text { Follow-up com } 16 \\
\text { semanas. }\end{array}$ & 3x semana. & $\begin{array}{l}\text { Treinamento muscular } \\
\text { inspiratório vs. treino } \\
\text { aeróbico. }\end{array}$ & $\begin{array}{c}\text { Threshold IMT1 } \\
\text { (Respironics) ou Power- } \\
\text { Breathe light or median } \\
\text { Resistance } \\
\text { (Powerbreathe) e } \\
\text { Cicloergômetro (Mini } \\
\text { Bike E5, ACTE Sports). }\end{array}$ & $\begin{array}{l}40 \text { minutos e } \\
15 \text { inspirações } \\
\text { para o TMI. }\end{array}$ & $\begin{array}{l}\text { Ambos os grupos } \uparrow \text { os parâmetros funcionais (capacidade } \\
\text { funcional, PImáx e força de membros inferiores) e biomarcadores } \\
\text { inflamatórios modulados, obtendo uma resposta semelhante ao } \\
\text { treinamento aeróbico de baixa intensidade. }\end{array}$ \\
\hline $\begin{array}{l}\text { MACAGNAN ET } \\
\text { AL. (2018) }\end{array}$ & $\begin{array}{l}4 \text { semanas com } \\
\text { intervalo de } 1 \\
\text { semana entre as } \\
\text { sessões. }\end{array}$ & 1x semana. & $\begin{array}{l}\text { Terapia de } \\
\text { fotobiomodulação. }\end{array}$ & $\begin{array}{l}\text { Intelect }{ }^{\circledR} \text { Advantage } \\
2766 \text { (Chattanooga Corp) } \\
\text { com uma sonda de } \\
\text { cluster composta por } \\
\text { cinco diodos e LASER } \\
\text { de } 850 \mathrm{~nm} / 200 \mathrm{~mW} \\
\text { (Chattanooga Corp). }\end{array}$ & $\begin{array}{l}\text { Não foi citado } \\
\text { o tempo. }\end{array}$ & $\begin{array}{l}\uparrow \text { de força significativa ocorreu após a aplicação de } \\
\text { fotobiomodulação, enquanto nenhuma alteração foi detectada } \\
\text { com placebo ou } 120 \mathrm{~J} / \text { braço. }\end{array}$ \\
\hline$\underset{(2018)}{\text { SUSUKI ET AL. }}$ & 8 semanas. & $3 x$ semana & Eletroestimulação muscular. & $\begin{array}{l}\text { Estimulador muscular } \\
\text { (Auto Tens Pro, Homer } \\
\text { Ion Co. Ltd). }\end{array}$ & 120 minutos. & $\begin{array}{c}\uparrow \text { da força e tamanho do músculo quadríceps, força extensora do } \\
\text { joelho. } \uparrow \text { em todos os componentes do SF-8, mas estes não foram } \\
\text { estatisticamente significativos. }\end{array}$ \\
\hline Yang et al. (2018) & $\begin{array}{l}3 \text { semanas }+ \\
\text { Follow-up com } 2 \text {, } \\
3,4 \text { e } 5 \text { semanas. }\end{array}$ & $3 x$ semana. & $\begin{array}{l}\text { Estimulação elétrica nervosa } \\
\text { transcutânea. }\end{array}$ & $\begin{array}{l}\text { TENS (D0207KL) e } \\
\text { eletrodos de carbono. }\end{array}$ & 20 minutos. & $\begin{array}{c}\text { Taxas de fluxo salivar } \uparrow \text { de forma estável e } \downarrow \text { da intensidade da } \\
\text { boca seca foi observada em todos os pós-testes. }\end{array}$ \\
\hline Ortí et al. (2018) & 4 semanas. & $3 x$ semana. & $\begin{array}{l}\text { Exercício de realidade virtual } \\
\text { vs exercícios convencionais } \\
\text { (aeróbicos + resistidos). }\end{array}$ & $\begin{array}{l}\text { Bolas, caneleiras e } \\
\text { bandas elásticas. } \\
\text { Programa ACT de } \\
\text { realidade virtual, } \\
\text { computador e televisão. }\end{array}$ & 30 minutos. & $\uparrow$ da função física em ambos os grupos. \\
\hline Testal et al. (2018) & 12 semanas. & $3 x$ semana. & $\begin{array}{l}\text { Exercícios resistidos } \\
\text { isométricos e aeróbicos vs } \\
\text { exercícios domiciliares. }\end{array}$ & Cicloergômetro. & 60 minutos. & $\begin{array}{c}\text { Nenhuma diferença estatisticamente significativa foi observada } \\
\text { nas doses e no rebote pós-diálise no grupo de exercício } \\
\text { intradialítico comparado aos domiciliares. }\end{array}$ \\
\hline
\end{tabular}




\begin{tabular}{|c|c|c|c|c|c|c|}
\hline Dong et al. (2019) & 12 semanas. & $3 \mathrm{x}$ semana. & Exercícios resistidos. & $\begin{array}{l}\text { Caneleiras e bola } \\
\text { elástica. }\end{array}$ & $\begin{array}{l}60 \text { a } 120 \\
\text { minutos. }\end{array}$ & $\begin{array}{c}\uparrow \text { do índice atividade física (força de preensão máxima, ritmo } \\
\text { diário e nível de atividade) e Kt / V, e } \downarrow \text { na proteína C reativa } \\
\text { foram encontrados entre os grupos I e C. Fatores inflamatórios IL } \\
\text {-6 e IL-10, e TNF- } \alpha \text { ) } \uparrow \text { ou } \downarrow \text { no grupo I. }\end{array}$ \\
\hline Silva et al. (2019) & 16 semanas. & $3 x$ semana. & Exercícios aeróbicos. & Cicloergômetro. & 30 minutos. & $\begin{array}{c}\uparrow \text { da vasodilatação mediada por fluxo e } \downarrow \text { na hipertrofia } \\
\text { ventricular esquerda e aldosterona sérica. Houve } \uparrow \text { da proteína C } \\
\text { reativa no GC. }\end{array}$ \\
\hline $\begin{array}{l}\text { Suhardjono et al. } \\
(2019)\end{array}$ & 12 semanas. & 2x semana. & $\begin{array}{l}\text { Exercícios aeróbicos vs. } \\
\text { exercícios aeróbicos e } \\
\text { resistidos. }\end{array}$ & $\begin{array}{l}\text { Cicloergômetro } \\
\text { (AdirMed®) e } \\
\text { Caneleiras. }\end{array}$ & 30 minutos. & $\begin{array}{l}\uparrow \text { na força das extremidades inferiores e na pontuação do } \\
\text { componente físico do instrumento KDQOL-SF TM, em ambos os } \\
\text { grupos de exercícios. }\end{array}$ \\
\hline Huang et al. (2020) & 24 semanas. & $3 \mathrm{x}$ semana. & $\begin{array}{l}\text { Exercício combinado } \\
\text { aeróbico e resistido. }\end{array}$ & Cicloergômetro. & 40 minutos. & $\begin{array}{c}\uparrow \mathrm{Kt} / \mathrm{V} \text { e da distância do TC6min, e } \downarrow \text { da pressão arterial } \\
\text { sistólica diastólica; mas não houve mudança significativa na } \\
\text { qualidade de vida. }\end{array}$ \\
\hline $\begin{array}{l}\text { Schardong et al. } \\
\text { (2020) }\end{array}$ & 8 semanas. & $3 \mathrm{x}$ semana. & Fotobiomodulação. & $\begin{array}{l}\text { LLL diodes (Thor® } \\
\text { Photomedicine, DD2). }\end{array}$ & $\begin{array}{l}\text { Não foi } \\
\text { informado. }\end{array}$ & $\begin{array}{l}\uparrow \text { da capacidade funcional para o GI, e da força muscular dos } \\
\text { membros inferiores. Para os outros resultados avaliados, não } \\
\text { houve diferença significativa. }\end{array}$ \\
\hline Yeh, et al. (2020) & 12 semanas. & $3 \mathrm{x}$ semana. & $\begin{array}{l}\text { Exercício combinado, } \\
\text { aeróbico e resistido. }\end{array}$ & $\begin{array}{l}\text { Cicloergômetro (Medical } \\
\text { Exercise Peddler 3000). }\end{array}$ & 30 minutos. & $\uparrow$ na distância do TC6min e no teste de sentar e levantar. \\
\hline $\begin{array}{l}\text { Yuenyongchaiwat et } \\
\text { al. (2020) }\end{array}$ & 8 semanas. & $3 x$ semana. & $\begin{array}{l}\text { Treinamento muscular } \\
\text { inspiratório. }\end{array}$ & $\begin{array}{l}\text { Threshold (Power } \\
\text { Breathe K5). }\end{array}$ & $\begin{array}{l}15 \text { inspirações } \\
\text { máximas, } 3 x, \\
\text { com } 60 \text { seg. de } \\
\text { repouso. }\end{array}$ & $\begin{array}{c}\uparrow \text { da força muscular inspiratória e da capacidade vital forçada. } \\
\text { Não houveram diferenças na qualidade de vida. }\end{array}$ \\
\hline Young et al. (2020) & 24 semanas. & $3 x$ semana. & $\begin{array}{l}\text { Exercício de ciclismo } \\
\quad \text { intradialítico. }\end{array}$ & Cicloergômetro. & 35-40 minutos. & $\begin{array}{l}\uparrow \text { a capacidade de realizar as atividades da vida diária e a } \\
\text { participação social foram resultados de primordial importância. }\end{array}$ \\
\hline
\end{tabular}

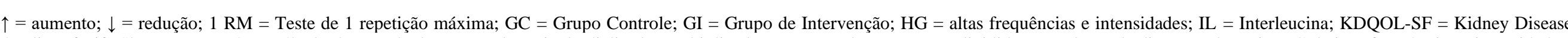

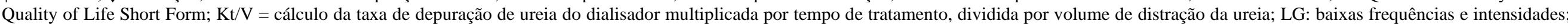

SF-8 = Short From-8; TC6min = Teste de Caminhada de 6 minutos; vs = versus. Fonte: Tabela desenvolvida pelos autores 
Tabela 3 - Classificação dos artigos conforme autor, ano, revista publicada, título do artigo, objetivo do estudo, metodologia adotada e resultados obtidos.

\begin{tabular}{|c|c|c|c|c|}
\hline Autor, Ano e Revista & Título & Objetivo & Metodologia & Resultados \\
\hline $\begin{array}{l}\text { Soto, Ortiz, Alvarenga } \\
\text { e Cuevas (2016) } \\
\text { Journal of Renal } \\
\text { Nutrition }\end{array}$ & $\begin{array}{l}\text { Effect of Resistance Exercises on the } \\
\text { Indicators of Muscle Reserves and } \\
\text { Handgrip Strength in Adult Patients on } \\
\text { Hemodialysis }\end{array}$ & $\begin{array}{l}\text { Avaliar o efeito } \quad \text { do } \\
\text { treinamento resistido em } \\
\text { pessoas em hemodiálise. }\end{array}$ & $\begin{array}{l}\text { Os pacientes foram randomizados para realizar } \\
\text { exercícios resistidos durante as sessões de } \\
\text { hemodiálise, com caneleiras e theraband. Os } \\
\text { exercícios foram realizados } 2 \text { x/semana, por } 12 \\
\text { semanas. O grupo controle recebeu apenas suas } \\
\text { sessões de hemodiálise. As medidas de desfecho } \\
\text { foram: circunferência muscular do braço e área } \\
\text { muscular do braço e dinamometria para medir a } \\
\text { força de preensão manual. }\end{array}$ & $\begin{array}{l}61 \text { pacientes sedentários em hemodiálise, com } \\
\text { idade média de } 29(21-39) \text { anos, sendo } 83 \% \text { com } \\
\text { algum grau de desnutrição. No GI: houve } \uparrow \text { da } \\
\text { circunferência muscular do braço de } 233,6(202- \\
254) \mathrm{mm} \text { para } 241,4(203-264) \mathrm{mm}(\mathrm{p}=0,001) \text {, a } \\
\text { área muscular do braço de } 35,9(26-41) \mathrm{cm} 2 \text { para } \\
36,6(26-46) \mathrm{cm} 2(\mathrm{p}=0,002) \text {, e a força de punho de } \\
19,6(11-28) \mathrm{kg} \text { para } 21,2(13-32) \mathrm{kg}(\mathrm{p}=0,05) \text {. }\end{array}$ \\
\hline $\begin{array}{l}\text { Roxo, et al. } \\
(2016) \\
\text { Journal Brazilian } \\
\text { Nephrology }\end{array}$ & $\begin{array}{l}\text { Impact of neuromuscular electrical } \\
\text { stimulation on functional capacity of } \\
\text { patients with chronic kidney disease on } \\
\text { hemodialysis. }\end{array}$ & $\begin{array}{l}\text { Avaliar os efeitos da } \\
\text { estimulação } \\
\text { neuromuscular na função } \\
\text { pulmonar e capacidade } \\
\text { funcional de pacientes com } \\
\text { doença renal crônica em } \\
\text { hemodiálise. }\end{array}$ & $\begin{array}{l}40 \text { adultos foram prospectivamente e randomizados } \\
\text { em dois grupos (controle } \mathrm{n}=20 \text { e tratamento } \mathrm{n}= \\
20 \text { ). O grupo intervenção recebeu estimulação } \\
\text { elétrica neuromuscular em quadríceps, } 3 \mathrm{x} / \mathrm{semana} \text {, } \\
\text { por } 30 \text { durante } 8 \text { semanas. Os desfechos avaliados } \\
\text { foram: espirometria, pressões respiratórias } \\
\text { máximas, teste de } 1 \mathrm{RM} \text { e teste da caminhada dos } \\
\text { seis minutos (TC6min), antes e após o período de } \\
\text { acompanhamento. }\end{array}$ & $\begin{array}{l}\mathrm{O} \text { grupo tratamento apresentou } \uparrow \text { da pressão } \\
\text { inspiratória máxima } \mathrm{p}=0,02 \text { na comparação entre } \\
\text { grupos e p }<0,001 \text { para a pressão máxima } \\
\text { expiratória. O teste de } 1 \mathrm{RM} \text { e a distância percorrida } \\
\text { no TC6min foram } \uparrow \text { no grupo de tratamento com } \\
\mathrm{p}<0,001 \text { e } 0,03 \text { respectivamente. Houve } \downarrow \text { da PAS } \\
(\mathrm{p}<0,001) \text { e FR }(\mathrm{p}<0,001) \text { após a estimulação } \\
\text { elétrica quando comparado ao grupo controle. }\end{array}$ \\
\hline $\begin{array}{c}\text { Bruggerman et al. } \\
\text { (2017) } \\
\text { Archives of Physical } \\
\text { Medicine and } \\
\text { Rehabilitation }\end{array}$ & $\begin{array}{l}\text { Effects of neuromuscular electrical } \\
\text { stimulation during hemodialysis on } \\
\text { peripheral muscle strength and exercise } \\
\text { capacity: a randomized clinical trial }\end{array}$ & $\begin{array}{l}\text { Avaliar os efeitos da } \\
\text { estimulação elétrica } \\
\text { neuromuscular de alta e } \\
\text { baixa frequência e } \\
\text { intensidade, na função física } \\
\text { e marcadores de inflamação } \\
\text { em pacientes com doença } \\
\text { renal crônica (DRC) em } \\
\text { hemodiálise. }\end{array}$ & $\begin{array}{l}51 \text { pacientes com DRC foram randomizados em } \\
\text { blocos de quatro por meio de envelopes opacos. } \\
\text { Eles foram divididos em um grupo de estimulação } \\
\text { elétrica neuromuscular de alta (HG) frequência } \\
(50 \mathrm{~Hz}) \text { e intensidade }(72,90 \mathrm{~mA}) \text { e um grupo de } \\
\text { baixa (LG) frequência ( } 5 \mathrm{~Hz}) \text { e intensidade } \\
(13,8 \mathrm{~mA})(\mathrm{LG}), 3 \text { vezes por semana durante uma } \\
\text { hora, durante } 12 \text { sessões (4 semanas). Foram } \\
\text { mensurados: força muscular periférica, capacidade } \\
\text { de exercício, níveis de músculo, marcador de } \\
\text { trofismo [fator de crescimento semelhante à } \\
\text { insulina tipo } 1 \text { (IGF-1) e níveis de pró-inflamatório } \\
\text { [fator de necrose tumoral (TNF-alfa) e anti- } \\
\text { inflamatório [Interleucina } 10 \text { - (IL-10) ] e citocinas. }\end{array}$ & 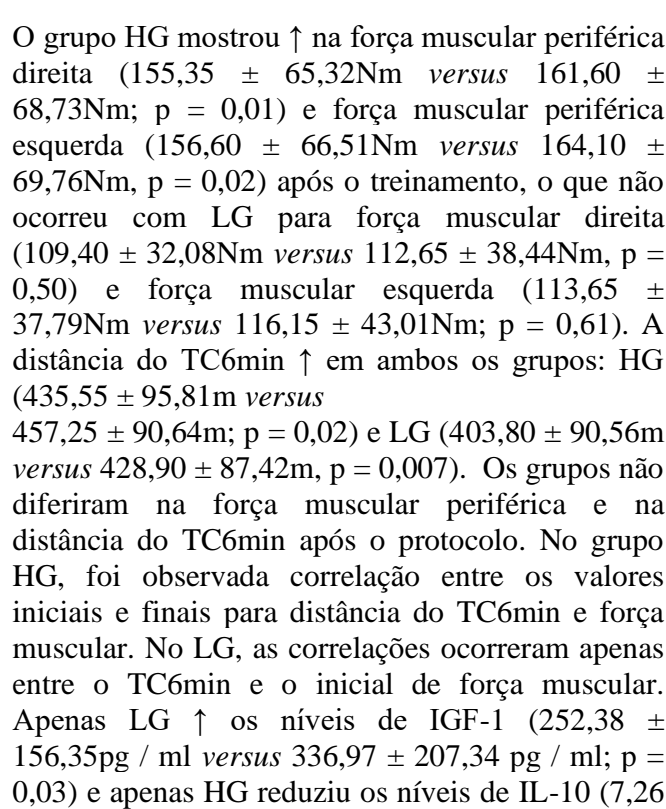 \\
\hline
\end{tabular}




\begin{tabular}{|c|l|}
\hline Álvares et al. (2017) & $\begin{array}{l}\text { Pneumatic compression, but not exercise, } \\
\text { can avoid intradialytic hypotension: a } \\
\text { American Journal } \\
\text { Nephrology }\end{array}$ \\
randomized trial.
\end{tabular}

Reduzir a hipotensão induzida pela hemodiálise e aumentar a remoção de fosfato usando ciclos de compressão pneumática.
Foram avaliados 21 pacientes e distribuídos aleatoriamente em grupos: controle; exercício de ciclismo durante os primeiros $60 \mathrm{~min}$; e compressão pneumática durante os primeiros $60 \mathrm{~min}$. Os dados obtidos incluíram impedância bioelétrica, medição de PA a cada hora, parâmetros bioquímicos, e quantificação de fosfato através do dialisado. A hipotensão foi definido como uma queda na média arterial pressão $(\mathrm{PAM}) \geq 20 \mathrm{~mm} \mathrm{Hg}$

$\pm 1,81 \mathrm{pg} / \mathrm{ml}$ versus $6,32 \pm 1,54 \mathrm{pg} / \mathrm{ml} ; \mathrm{p}=0,03)$. Os grupos não mostraram diferenças nas concentrações de TNF-alfa.

Não houve diferença na taxa de ultrafiltração $(\mathrm{p}=$ $0,628)$, peso delta $(\mathrm{p}=0,415)$, delta de água corporal total, intra e extracelular entre os grupos controle, ciclismo e compressão pneumática $(p=0,209, p=0,348$ e $p=0,467$ respectivamente). Delta MAP foi alterado por compressão pneumática quando comparado com controle, ciclagem e a compressão pneumática, respectivamente $(-4,7$ [$17,2,8,2],-4,7[-20,5,-0,2]$ e $-2,3[-8,1,9,0]$ $\mathrm{mmHg} ; p=0,021)$. A hipotensão induzida por hemodiálise ocorreu em 43, 38 e $24 \%$ dos pacientes em controle, ciclismo e compressão pneumática, respectivamente $(p=0,014)$. A remoção de fosfato não aumentou em qualquer intervenção $(p=0,486)$. A maior remoção de fosfato foi dependente na ultrafiltração, fosfato sérico pré-diálise e hormônio da paratireoide mais alto.

\begin{tabular}{c|l|l|}
\hline Schardong et al. (2017) & Effects of intradialytic neuromuscular & Avaliar os efeitos da \\
Artificial Organs - & electrical stimulation on strength and estimulação e elétrica \\
Wiley Online Library & muscle architecture in patients with neuromuscular em pacientes \\
& $\begin{array}{l}\text { chronic kidney failure: randomized } \\
\text { clinical trial }\end{array}$ & $\begin{array}{l}\text { com insuficiência renal } \\
\text { crônica em hemodiálise. }\end{array}$
\end{tabular}

21 pacientes foram randomizados em um grupo controle ( $n$ 510) e grupo de estimulação elétrica neuromuscular ( $n$ 511) que recebeu EENM em quadríceps por 8 semanas, 3 vezes/semana (20-34 min). A força muscular dos membros inferiores foi avaliada por dinamometria e teste de sentar e levantar (TSL); a arquitetura muscular por ultrassonografia; capacidade funcional pela distância percorrida em um TC6min; e a função endotelial pela técnica de dilatação mediada por fluxo da artéria braquial.

Investigar o efeito do Foram randomizados pacientes em hemodiálise de

Cho et al. (2018) International Urology and Nephrology
Effect of intradialytic exercise on daily physical activity and sleep quality in maintenance hemodialysis patients atividade física dínia e na qualidade do sono, medida por um acelerômetro, em pacientes em hemodiálise de manutenção
Houve $\uparrow$ na força de membros inferiores na EENM em comparação com o GC (dinamometria: 43,286 16,94 vs. $35,84616,89, \mathrm{p}=5$ 0,006;

TSL: 16.1066 .51 vs. $12.5064 .7, p=50.029)$. Havia uma $\downarrow$ nos ângulos de penetração do vasto lateral direito e vasto lateral esquerdo no CG quando comparado ao EENM (VLD: 11,93 [10,70-15,11] vs. $13,57[11,81-15,96], p=50,039$; VLE: 11,62 [9,00-14,20] vs. $15,52[12,86-20,02], p=50,042$ ). Não houve mudança em capacidade funcional e na função endotelial para CG e EENM, respectivamente.

Observou-se um $\uparrow$ no equivalente metabólico MET; $\mathrm{kcal} / \mathrm{h} / \mathrm{kg})$ no EA $(1,02 \pm 0,03$ vs $1,04 \pm$ $0,04, p=0,04)$ e EC $(1,06 \pm 0,05$ vs $1,09 \pm 0,08, P=$ $0,01)$ em 12 semanas em comparação com a linha de base. Ao comparar mudanças entre os grupos em MET, houve um $\uparrow$ no grupo EC $(0,03 \pm 0,03$ vs $0,01 \pm 0,04, p=0,02)$ em comparaça com o grupo controle. O número total de episódios sedentários ergométrica, TheraBand $₫ /$ theraball. Realizaram 
exercícios intradialíticos por 12 semanas (3 vezes/semana). $\mathrm{O}$ grupo controle $(\mathrm{n}=13)$ recebeu apenas alongamentos. No início do estudo e acompanhamento de 12 semanas, a atividade física diária e a qualidade do sono foram medidos com um acelerômetro triaxial (wActiSleepBT;ActiGraph,Pensacola, FL) durante um período contínuo de 7 dias. (por semana) diminuiu significativamente no EA $(200 \pm 37$ vs $174 \pm 36, P=0,01)$, ER $(180 \pm 31$ vs $130 \pm 49, P=0,03)$ e EC $(180 \pm 45$ vs $152 \pm 46, P$ $=0,04)$ em 12 semanas em comparação com o valor basal. O índice médio de fragmentação do sono $\downarrow$ em comparação com a linha de base nos grupos EA $(51,4 \pm 8,0$ vs $44,5+9,6, p=0,03)$ e $\operatorname{ER}(52,3 \pm 7,3$ vs $40,0 \pm 15,4, p=0,01$.

Rosa et al., (2018) Effect of continuous progressive Investigar o efeito do 52 pacientes em hemodiálise (idade 55,7 $\pm 14,03$ Clinical Rehabilitation patients: a randomized controlled trial resistance training during hemodialysis on body composition, physical function and quality of life in end-stage renal disease

te resistência progressiva contínua sobre composição corporal, capacidade funcional qualidade de vida auto referida em pacientes renais em estágio terminal.

anos) foram randomizados para exercício

(treinamento de resistência progressiva (TRP, $n=$

28) ou grupo controle (CON, $n=24)$. Os pacientes randomizados para o grupo TRP realizaram exercícios de força (2 séries de 15-20 repetições), três vezes por semana durante 12 semanas. Os pacientes randomizados para o grupo $\mathrm{CON}$ receberam um exercício simulado com mobilização de braços e pernas sem carga e progressão. Foram mensuradas: composição corporal usando absorciometria de raio-X de dupla energia, força usando dinamometria de preensão manual, teste sitto-stand repetido, TC6min, flexibilidade e o questionário SF-36 (qualidade de vida (QV)) foram avaliados no início e em 12 semanas.

Figueiredo et al (2018) ' Effects of the inspiratory muscle traing PLoS ONE Effects of the inspiratory muscle training and aerobic training on respiratory and functional parameters, inflammatory biomarkers, redox status and quality of life in hemodialysis patients: randomized clinical trial
Avaliar e comparar os 37 pacientes foram randomizados com alocação efeitos isolados e fatorial e análise de intenção de tratar para realizar combinados do treinamento 8 semanas de TMI a $50 \%$ da pressão inspiratória muscular inspiratório (TMI) máxima (PImáx), TA de baixa intensidade ou e Treinamento Aeróbico (TA) sobre parâmetros respiratórios e funcionais, marcadores

bioinflamatórios, estado redox e qualidade de vida relacionada à saúde (QVRS) em pacientes em hemodiálise.
Massa magra da perna $(p=0,04))$, conteúdo mineral ósseo $(\mathrm{p}=0,02)$,

força da perna em repetições do teste sit-to-stand $(\mathrm{p}=0,01)$ e flexibilidade $(p<0,01)$ foram significativamente $\uparrow$ no grupo TRP em comparação com o CON. Capacidade de caminhada, força muscular periférica e qualidade de vida não eram diferentes entre os grupos.

Foram observados $\uparrow$ de PImáx, capacidade funcional, força de membros inferiores e níveis de resistina e $\downarrow$ dos níveis de sTNFR2 em 16 semanas, em comparação com a linha de base e 8 semanas, foram observados em todos os grupos $(p<0,001)$. TMI $\uparrow$ a capacidade funcional, PImáx e força de membros inferiores em 96,7m (IC95\% 5,6-189,9), $34,5 \mathrm{cmH} 2 \mathrm{O}$ (IC95\% 22,4-46,7) e 2,2 repetições (IC95\% 1,1-3,2) respectivamente. $\uparrow$ nos níveis de resistina e $\downarrow$ nos níveis de sTNFR2 após IMT foi de $0,8 \mathrm{ng} / \mathrm{dL}$ (IC95\% 0,5-1,1) e 0,8ng/dL (IC95\% 0,3 $1,3)$, respectivamente, sem diferença entre os grupos. Em comparação com a linha de base e 8 semanas, os níveis de adiponectina $(\mathrm{p}<0,001)$ e o domínio de fadiga da QVRS ( $<<0,05)$ aumentou em 16 semanas apenas no TC. TMI, TA e TC $\uparrow$ os parâmetros funcionais e biomarcadores
(sTNFR1) e 2 (sTNFR2), adiponectina, 
resultados secundários. As análises de dados foram realizadas de duas vias medições repetidas ANOVA. \begin{tabular}{c|l} 
Macagnan et al. (2018) & Acute effect of photobiomodulation \\
Lasers in Medical & therapy on handgrip strength of chronic \\
Science & kidney disease patients during
\end{tabular} hemodialysis.
Avaliar o efeito agudo de três diferentes doses de fotobiomodulação na força de preensão manual máxima de pacientes com DRC.
15 voluntários ( $58 \pm 8$ anos, 10 homens/5 mulheres) em hemodiálise crônica $(6 \pm 4$ anos) participaram de um estudo randomizado, cruzado, duplo-cego, controlado por placebo. Cada paciente foi avaliado em 4 quatro sessões, com intervalo de 1 semana entre as avaliações. Placebo ou fotobiomodulação (sonda de cluster com cinco $850 \mathrm{~nm} /$ Diodos laser de $200 \mathrm{~mW}$ ) foram aplicados em três locais ao longo dos flexores do dedo (doses totais de 60, 90 ou $120 \mathrm{~J}$ por braço). O a força máxima de preensão manual foi avaliada antes e depois do tratamento com fotobiomodulação ou placebo em cada sessão.

$\begin{array}{cll}\begin{array}{c}\text { Susuki et al. (2018) } \\ \text { Artificial Organs }\end{array} & \begin{array}{l}\text { Beneficial effect of intradialytic electrical } \\ \text { muscle stimulation in hemodialysis } \\ \text { patients: a randomized controlled trial. }\end{array} & \begin{array}{l}\text { eletroestimulação muscular } \\ \text { (EEM) intradialítica. }\end{array}\end{array}$
patients: a randomized controlled trial.
29 pacientes foram aleatoriamente designados para o grupo EEM ou o grupo de controle (sem treinamento), 13 pacientes em cada grupo foram eventualmente analisados. O grupo de intervenção recebeu EEM intradialítico durante um período de 8 semanas. Foi realizada medida de força isométrica de extensores de joelho usando um dinamômetro de mão momento, avaliação da área transversa do quadríceps usando imagem de ressonância magnética (MRI), o Teste Timed Up \& Go (TUG) para avaliação da função física, a versão japonesa do Short Form-8 Health Pesquisa (SF-8), e exames de sangue foram realizados antes e após o período de intervenção.

inflamatórios modulados, além disso, o TMI provocou uma resposta semelhante ao TA de baixa intensidade em pacientes em hemodiálise.

$\uparrow$ de força significativos ocorreram após a aplicação de fotobiomodulação com doses de $60 \mathrm{~J} /$ braço $(4,85 \%, p=0,005)$ e $90 \mathrm{~J} /$ braço $(4,45 \%, p=$ 0,013 ), enquanto nenhuma alteração foi detectada com placebo ou $120 \mathrm{~J} / \mathrm{braço}$

Os resultados foram melhora do músculo quadríceps, em força e tamanho, respectivamente. O grupo EEM demonstrou melhoria significativa em comparação com o grupo controle em termos de força extensora do joelho (direita: 22,36 12,8 vs. $210,8622,3 \mathrm{~N}, p<0,001$; esquerda: $26,1629,7$ vs $28,3618,7 \mathrm{~N}, \quad p<0,001)$, área transversa do quadríceps em três posições, 25,50 e $75 \%$ do comprimento do segmento do trocânter maior para borda inferior da epicôndilo lateral do fêmur (25\% à direita: grupo EEM 1,76 2,0 vs. Grupo controle $20,461,8 \mathrm{~cm} 2, p=0,05 ; 25 \%$ esquerda: grupo EEM 1,36 1,1 vs. grupo controle 20,66 1,8 $\mathrm{cm} 2, P=0,01 ; 50 \%$ direito: grupo EEM 2,06 2,2 vs. grupo controle $20,761,9 \mathrm{~cm} 2, P=0,004 ; 50 \%$ esquerdo: grupo EEM 2.762.1 vs. Grupo Controle

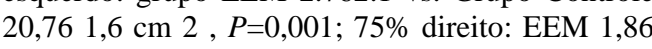
2,2 vs. grupo controle $20,761,5 \mathrm{~cm} 2, P=0,003$; $75 \%$ esquerdo: grupo EEM 2,16 1,9 vs. grupo controle $20.461 .5 \mathrm{~cm} 2, P=0,003)$; e tempo TUG ($0,860,6$ vs. $0,260,5$ s, $P<0,001)$.

The effect of transcutaneous electrical nerve stimulation (TENS) on increasing

Yang, Chen, Su e Chi (2018) Oral Disease salivary flow rate in hemodialysis patients.
Avaliar o impacto de um programa de estimulação elétrica nervosa transcutânea (TENS) n

(

Um estudo simples-cego selecionou 80 indivíduos que foram atribuídos aleatoriamente a um grupo de ratamento que recebeu TENS de $50 \mathrm{~Hz}$ e $250 \mu \mathrm{s}$ e um grupo de controle recebendo TENS à $2 \mathrm{~Hz}$ e 50
Após a conclusão de 6 sessões de TENS, todas as taxas de fluxo salivar $\uparrow$ de forma estável até o final de 9 sessões para o grupo de tratamento e na semana de acompanhamento após o tratamento. No 
sensação de boca seca e taxas de fluxo salivar de pacientes em hemodiálise.
Ortí et al. (2018) Virtual Reality Exercise Intradialysis to Scandinavian Journal Improve Physical Function: A Feasibility of Medicine \& Science Randomized Trial. Sports
Avaliar a viabilidade da 18 indivíduos foram randomizados para um realização de uma programa de exercícios combinados de intradiálise intervenção de exercício de de 16 semanas. O programa durou 4 semanas, de realidade virtual (RV) não exercícios combinados ou exercícios de realidade imersiva intradialítica. $\mathrm{O}$ virtual. As medidas avaliativas principais foram: objetivo secundário era função física, medida através de vários testes explorar o impacto dos confiáveis (testes de sentar e levantar 10 e 60, exercícios convencionais ou velocidade de marcha, testes de elevação do de RV na função física. calcanhar de uma perna e TC6min.

Testal et al. (2018) Influence of Physical Exercise on the Therapeutic Apheresis Dialytic Adequacy Parameters of Patients and Dialysis

69 pacientes randomizados em um grupo de primeiras 2 horas de sessões de hemodiálise e outro
Avaliar se a realização exercícios físicos em hemodiálise crônica poderia melhorar a dose de diálise e reduzir o rebote pós-diálise. grupo que realizou exercício físico domiciliar As variáveis descritivas foram a doses de diálise medidas por $\mathrm{Kt} / \mathrm{V}$ e rebotes (uréia, creatinina, potássio, fósforo), foram registrados. O Kt/V médio foi de 1,84 ; o rebote de 30 minutos da creatinina foi de $32,37 \%$, uréia $24,39 \%$, potássio $15,31 \%$ e fósforo $51,29 \%$. Para cada paciente, a medição basal foi comparada com aquelas determinadas quando realizados exercícios físicos.

\begin{tabular}{c|l|l|}
\hline $\begin{array}{c}\text { Dong, Zhangl e Yin } \\
\text { (2019) }\end{array}$ & $\begin{array}{l}\text { Effects of intradialytic resistance exercise } \\
\text { on systemic inflammation in maintenance }\end{array}$ & $\begin{array}{l}\text { Investigar o exercício de resio do } \\
\text { hemodialysis patients with sarcopenia: a }\end{array}$ \\
$\begin{array}{c}\text { International Urology } \\
\text { and Nephrology }\end{array}$ & $\begin{array}{l}\text { mancadores de inflamação e } \\
\text { randomized controlled trial }\end{array}$ & $\begin{array}{l}\text { indices de sarcopenia em } \\
\text { pacientes em hemodiálise } \\
\text { de manutenção com }\end{array}$ \\
& & sarcopenia.
\end{tabular}

41 em hemodiálise e com sarcopenia foram divididos em um grupo de intervenção (grupo I, $n=$ 21) e um grupo controle (grupo $\mathrm{C}, n=20$ ). Os pacientes do grupo $\mathrm{C}$ receberam apenas cuidados de hemodiálise de rotina, enquanto os pacientes do grupo I receberam exercício de resistência intradialítica progressiva com intensidade alta ou us nos pontos de acupuntura ST 6 e TE1, $3 \mathrm{x} /$ semana, por 3 semanas. A taxa de fluxo salivar total e a intensidade da boca seca foram medido totalmente cinco vezes para ambos os grupos, no pré-tratamento, após 3, 6, 9 sessões de TENS e uma semana após o término do tratamento. pacientes que realizou exercício físico durante as

entanto, a melhora significativa na intensidade da boca seca foi observada em todos os pós-testes do que no pré-tratamento em ambos os grupos.

Houve um efeito de tempo significativo, de modo que a função física $\uparrow \mathrm{em}$ ambos os grupos. A adesão não foi significativamente diferente entre os grupos moderada, por 12 semanas, $3 \mathrm{x} /$ semana (usando o peso dos membros inferiores e movimento da bola elástica do membro superior) com base nos cuidados de hemodiálise de rotina
Após 12 semanas, uma diferença significativa no status de atividade física (força de preensão máxima, ritmo diário e nível de atividade), Kt/V e grupos I e C. Fatores inflamatórios (interleucina (IL) -6, a IL-10 e o fator de necrose tumoral (TNF) $-\alpha) \uparrow$ ou $\downarrow$ mais significativamente no grupo I do que no grupo $\mathrm{C}$. proteína $\mathrm{C}$ reativa foram encontrados entre os

Nenhuma diferença estatisticamente significativa foi observada entre as alterações determinadas para dose de rebote no grupo com exercícios intradialíticos em comparação com aqueles do grupo de exercícios em casa.

30 pacientes participaram do estudo que foi um

No IG, houve uma melhora estatisticamente 
Cardiorenal Medicine nontraditional cardiovascular risk factors treinamento aeróbico não in hemodialysis patients: results from a tradicional nos fatores de prospective randomized trial risco cardiovascular em ensaio prospectivo, controlado e randomizado com análise de intenção de tratamento. Estes foram pacientes com DRC em hemodiálise. rigidez arterial, ecocardiograma, análise de ubmetidos a teste ergométrico, avaliação de reatividade endotelial, ultrassom de carótida e testes laboratoriais, incluindo análise de aldosterona sérica. O grupo de intervenção (GI) $(n=15)$ foi submetido à exercício aeróbico durante a hemodiálise, $3 \mathrm{x} /$ semana, durante 4 meses. $\mathrm{O}$ grupo de controle (GC) $(n=15)$ não recebeu intervenção. Todos os pacientes foram reavaliados após 4 meses.

Suhardjono, Umami e I The effect of intradialytic exercise twice a 120 pacientes foram randomizados aleatoriamente
Tedjasukman (2019) Hemodialysis International week on the physical capacity, inflammation, and nutritional status of

Determinar o papel do exercício intradialítico 2 vezes por semana na

inflamação nutricional em pacientes em diálise e determinar quais exercícios são mais adequados para essa

população.

20 pacientes foram randomizados aleatoriamente em grupos de 1 de 3 , ou seja, um grupo de pacientes realizando exercícios aeróbicos, um grupo de pacientes realizando exercícios combinados e o grupo controle. $\mathrm{O}$ estudo foi realizado por 12 emanas de fevereiro a maio de 2018.

Investigar os efeitos do No total, 47 indivíduos elegíveis foram alocados exercício combinado de na aleatoriamente para o grupo de exercícios e o grupo eficiência da hemodiálise, de controle. O grupo de intervenção realizou pressão sanguínea, exercícios combinados intradialíticos de 24 capacidade de exercício e semanas, 3x/semana, em intensidade moderada. $\mathrm{O}$ qualidade de vida em grupo controle recebeu os cuidados habituais. O pacientes em hemodiálise. desfecho primário era a eficiência da hemodiálise, que era registrada a cada 4 semanas. E o secundário era a pressão arterial, capacidade de exercício e qualidade de vida, medida no início do estudo e após 24 semanas de intervenção

\begin{tabular}{c|l|}
\hline Schardong et al. (2020) & Photobiomodulation therapy increases \\
Lasers in Medical & functional capacity of patients with \\
Science & $\begin{array}{l}\text { chronic kidney failure: randomized } \\
\text { controlled trial. }\end{array}$
\end{tabular}

Avaliar o efeito crônico da Um ensaio controlado e randomizado foi realizado fotobiomodulação na com um grupo de intervenção (GI, $n=14$ ) que capacidade funcional e, recebeu 24 sessões de fotobiomodulação $(810 \mathrm{~nm}, 5$ secundariamente, investigar diodos $\times 200 \mathrm{~mW}, 30 \mathrm{~J} /$ local de aplicação) no seus efeitos do na força, membro inferior durante a hemodiálise. $\mathrm{O}$ grupo espessura muscular e controle $(\mathrm{GC}, n=14)$ não recebeu intervenção ecogenicidade, percepção fisioterapêutica. significativa na vasodilatação mediada por fluxo FMV; $p=0,002)$ e uma $\downarrow$ na hipertrofia ventricular esquerda $(\mathrm{p}=0,006)$ e na aldosterona sérica $(p=$ $0,016)$. Houve $\uparrow$ da proteína $\mathrm{C}$ reativa no $\mathrm{GC}(\mathrm{p}=$ $0,002)$

Houve um significativo $\uparrow$ na força das extremidades inferiores no grupo que realiza exercícios aeróbicos e nos exercícios combinados, em comparação com o grupo controle. Houve também um significativo $\uparrow$ na pontuação do componente físico do instrumento KDQOL-SF no treinamento aeróbio e nos grupos de exercícios combinados. Não houveram diferenças significativas entre $o$ grupo de exercícios combinados e o grupo de treinamento aeróbio em qualquer resultado.

No grupo de intervenção, a Kt / V melhorou significativamente em $13,2 \%$, e a pressão arterial sistólica e a pressão arterial diastólica significativamente em $8,5 \mathrm{mmHg}$ e $6,5 \mathrm{mmHg}$, respectivamente. A distância do TC6min $\uparrow$ por 43 m $(9,8 \%)$, mas não houve mudança significativa na qualidade de vida.

Como resultado, houve $\uparrow$ da capacidade funcional (avaliada por meio do TC6min) para o GI em comparação com o GC [50,7 m (IC95\% 15,63; $85,72), \mathrm{p}=0,01$, tamanho do efeito grande, $\mathrm{d}=$ $1,12]$, bem como $\uparrow$ na forca muscular dos membros inferiores (avaliada por meio do teste de sentar e levantar) [- 7,4 s (IC95\% - 4,54; - 10,37), p = 0,00, 
da dor, fadiga e qualidade

de vida dos pacientes. tamanho do efeito grande, $d=1,99]$. Para os outros resultados avaliados, não foi observada diferença significativa entre os grupos.

\begin{tabular}{|c|c|c|c|}
\hline $\begin{array}{l}\text { Yeh, Wang, Hsu e Liu } \\
(2020) \\
\text { Clinical Rehabilitation }\end{array}$ & $\begin{array}{l}\text { Twelve-week intradialytic cycling } \\
\text { exercise improves physical functional } \\
\text { performance with gain in muscle strength } \\
\text { and endurance: a randomized controlled } \\
\text { trial. }\end{array}$ & $\begin{array}{lr}\text { Avaliar o } & \text { efeito do } \\
\text { exercício de } & \text { ciclismo } \\
\text { intradialítico } & \text { no } \\
\text { desempenho } & \text { físico } \\
\text { funcional com ganho de } \\
\text { força e resistência muscular } \\
\text { em pacientes renais em fase } \\
\text { terminal em hemodiálise. }\end{array}$ & $\begin{array}{l}76 \text { pacientes regulares em hemodiálise, recrutados e } \\
\text { igualmente aleatorizados para o grupo de exercícios } \\
\text { e grupos de controle, com medições realizadas em } \\
4,8 \text { e } 12 \text { semanas de exercício de ciclismo } \\
\text { intradialítico. O exercício de ciclismo intradialítico } \\
\text { foi realizado por } 12 \text { semanas e compreendeu as } \\
\text { fases de aquecimento, principal e desaquecimento. } \\
\text { Foi utilizado um equipamento de ciclismo } \\
\text { estacionário, que envolve as modalidades aeróbica } \\
\text { e de resistência. A intensidade foi mantida com um } \\
\text { pouco de esforço. Os exercícios eram realizados por } \\
30 \text { minutos, com início na segunda hora de } \\
\text { tratamento. Os resultados medidos foram a } \\
\text { distância do TC6min, tempo necessário para } \\
\text { completar } 10 \text { ciclos do teste de sentar e levantar e } \\
\text { número de repetições em } 60 \text { segundos. }\end{array}$ \\
\hline
\end{tabular}

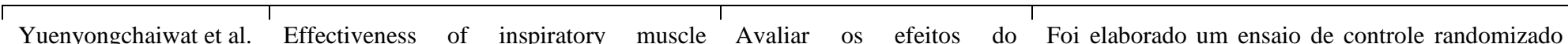

(2020) training on respiratory fitness and

Physiotherapy breathlessness in chronic renal failure: A Research International randomized control trial.

\section{Avaliar os efeitos do} inspiratório em pacientes em hemodiálise sobre aptidão respiratória, qualidade de vida e falta de ar.
A idade média (desvio padrão) dos participantes foi de 55,47 (13,00) anos. A distância do TC6min foi significativamente diferente nas semanas $8(P$ $=0,01)$ e $12(P<0,001)$ no grupo de exercício em comparação com o grupo controle no início do estudo. Notavelmente, os resultados do teste de sentar e levantar $(P=0,01) \quad$ influenciaram significativamente a distância do TC6min. O resultado teste de sentar e levantar $\uparrow$ significativamente no grupo de exercícios $(P<0,05)$. com 50 pacientes que realizaram hemodiálise $(25$ indivíduos em cada grupo; treinamento muscular inspiratório e grupo controle). Função pulmonar, força de RM, QV (medido por Kidney Disease Quality of Life-36) e sensação de falta de ar foram medidos antes e após uma intervenção de 8 semanas.

A força muscular inspiratória $\uparrow$ no grupo de intervenção após um programa de 8 semanas $(\Delta 25,92 \pm 8,73 \mathrm{cmH} 2 \mathrm{O}, \mathrm{p}=0,005)$. Valores significativamente $\uparrow$ de músculos inspiratórios e capacidade vital forçada no grupo de treinamento foram observados após uma intervenção de 8 semanas $(\Delta 12,44 \pm 3,07 \mathrm{cmH} 2 \mathrm{O}$ e $\Delta 0,097 \pm 0,046$ $\mathrm{L}$, respectivamente), mas não no grupo controle. Nem o grupo de treinamento, nem o grupo controle houveram mudanças na QV. No entanto, a sensação de falta de ar $\downarrow$ no grupo de treinamento muscular inspiratório, mas não no grupo controle.

\begin{tabular}{c|l}
\hline $\begin{array}{c}\text { Young et al. (2020) } \\
\text { BMJ Open }\end{array}$ & $\begin{array}{l}\text { Exercise for people living with frailty and } \\
\text { receiving haemodialysis: a mixed methods } \\
\text { randomised controlled feasibility study. }\end{array}$
\end{tabular}

Determinar exercícios Pacientes adultos em hemodiálise prevalentes do intradialíticos são viáveis para pacientes frágeis em hemodiálise, e explorar como a intervenção pode ser adaptada às suas necessidades ensaio CYCLE-HD com uma pontuac̃a da escala de fragilidade clínica de 4-7 (vulneráveis a gravemente frágeis) eram elegíveis para o estudo de viabilidade. Os participantes do grupo de exercícios realizaram 6 meses, 3x/semana, ciclismo intradialítico (CID) progressivo de intensidade moderada.
De 124 pacientes elegíveis, 51 (80\%) completaram avaliação inicial. $\mathrm{n}=24$ dialisados durante turnos designados aleatoriamente para exercícios e $\mathrm{n}=27$ $6(12 \%)$ foram perdidos no acompanhamento. O grupo de exercícios completou $74 \%$ das sessões. 27\%-89\% dos dados de resultados secundários faltaram. Pacientes frágeis em hemodiálise descreveram várias maneiras de aprimorar os em turnos designados para cuidados habituais. $\mathrm{n}=$ 
procedimentos do estudo. Manter a capacidade de social foram resultados de primordial importância. Os participantes desejavam um programa de exercícios variado.

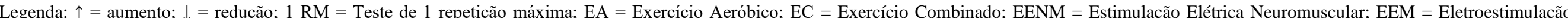

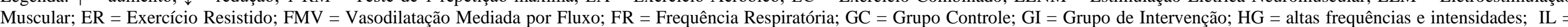

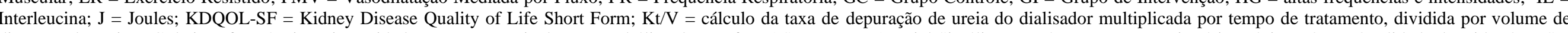

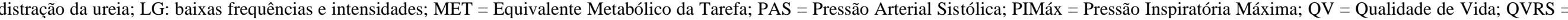

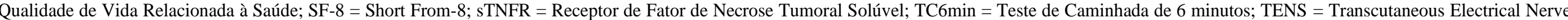

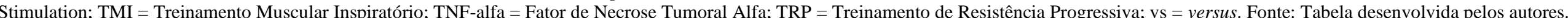




\section{Discussão}

A reabilitação intradialítica tem demonstrado importantes resultados em diferentes marcadores quando refere-se aos pacientes renais crônicos que passam diariamente por terapias substitutivas invasivas, como a hemodiálise, por diversas horas consecutivas. Com isso, diversos estudos têm discutido acerca da aplicação de um programa intradialítico de exercícios a fim de melhorar aspectos físicos e psicossociais destes pacientes (Santos et al., 2017). Sendo encontrados através de exercícios resistidos, aeróbicos, combinados, entre outras modalidades, a melhora de fatores relacionados à força muscular, capacidade de exercício físico, capacidade funcional, entre outros desfechos.

Uma das modalidades de exercício utilizadas são os exercícios aeróbicos, que refletem na modificação de diversos sistemas fisiológicos do corpo humano, principalmente em parâmetros cardíacos, pulmonares e vasculares, visto seu elevado nível de consumo de oxigênio (Júnior et al., 2021). Sabe-se que as doenças cardiovasculares e cardiopulmonares representam um importante fator de risco aos pacientes renais crônicos, fator este que auxilia na classificação e na indicação do manejo clínico do paciente com DRC, conforme seu estágio (Brasil, 2014).

O estudo de Silva et al. (2019) trouxeram a aplicação de um programa de exercícios aeróbicos a fim de analisar possíveis alterações em fatores de risco cardiovasculares. O programa consistia em atividade aeróbica através de cicloergômetro estacionário por trinta minutos, três vezes por semana, em um período de dezesseis semanas totais. Efeitos positivos foram encontrados, como o aumento da vasodilatação fluxo-mediada, redução da hipertrofia do ventrículo esquerdo e dos níveis de aldosterona sérica. E ainda, o grupo que não recebeu intervenção teve um aumento dos níveis de proteína C reativa sanguínea, o que indica o desenvolvimento ou aumento de processos inflamatórios ou infecciosos, o que não foi encontrado no grupo que recebeu a reabilitação.

A literatura nos traz que os exercícios aeróbicos estão diretamente relacionados às modificações cardiovasculares, além da melhora da função física. Porém sua real efetividade está diretamente relacionada a intensidade a qual esta modalidade de exercício é realizada. Exercícios aeróbicos realizados em intensidades moderadas a altas podem realmente refletir na redução de processos infecciosos e inflamatórios, na melhora da função endotelial e na redução do risco de hipertensão arterial (Waclawovsky et al., 2021; Powers \& Howley, 2014)

Young et al. (2020) avaliaram também se o exercício aeróbico por cicloergômetro poderia trazer resultados positivos relacionados ao estado de fragilidade dos pacientes em hemodiálise de manutenção. Foi realizado um estudo de vinte e quatro semanas, com exercícios de tempo variável de trinta e cinco a quarenta minutos, encontrando um aumento na capacidade de realizar atividades básicas de vida diária e também da participação social, contribuindo positivamente aos pacientes. Mas foi relatado pela população do estudo, ao final, o desejo de um programa de exercícios mais variado.

Soto et al. (2016), a fim de avaliar o efeito do exercício resistido intradialítico em pacientes com DRC, aplicaram um programa de cinquenta minutos de exercícios através de caneleiras e bandas elásticas, duas vezes na semana, por doze semanas. Tal programa resultou em um aumento da circunferência muscular e da área muscular do braço e da força de punho, refletindo positivamente na massa e na força muscular desses pacientes.

Corroborando aos efeitos positivos do exercício resistido, Rosa et al. (2018) avaliaram por meio de comparação entre grupo controle e intervenção os exercícios resistidos progressivos na composição corporal, capacidade funcional e na qualidade de vida dos pacientes em fases terminais da DRC. Os exercícios foram realizados três vezes por semana, com tempo de quarenta a cinquenta minutos, realizados com pesos livres e bandas elásticas, por doze semanas. Pôde-se observar aumento da massa magra e força de membros inferiores em repetições, aumento do conteúdo mineral ósseo, e da flexibilidade. Porém não foi possível afirmar a melhora da capacidade funcional, força muscular periférica e da qualidade de vida. Isso pode ser justificado pois apenas um treinamento de resistência isolado não seria capaz de reabilitar completamente o indivíduo, dependendo tais variáveis de mais intervenções, além desta modalidade de treinamento única, sendo por exemplo, a melhora 
da capacidade funcional completamente dependente da realização do exercício aeróbico, através do aprimoramento de fatores cardiopulmonares (Rosa et al., 2018; Orcy et al., 2011).

Dong et al. (2019), buscaram investigar o efeito dos exercícios resistidos nas alterações dos marcadores inflamatórios e em índices de sarcopenia nesses pacientes através de um programa de reabilitação de doze semanas, com exercícios três vezes na semana através de caneleiras e bolas com duração variável de sessenta a cento e vinte minutos. Foi observado um aumento do índice de atividade física através da força de preensão máxima manual e da taxa de Kt/V, que serve para manejar a hemodiálise em dose e qualidade. $\mathrm{O}$ aumento desta taxa pelo programa de exercícios físicos poderá refletir positivamente em índices de morbimortalidades de pacientes com DRC em hemodiálise de manutenção (Breitsameter et al., 2012). Além disso, o exercício resistido também ocasionou uma redução do índice de proteína $C$ reativa nos pacientes. Fatores inflamatórios interleucina-6 (IL-6), interleucina-10 (IL-10) e o fator de necrose tumoral (TNF- $\alpha$ ) aumentaram ou diminuíram no grupo de intervenção. Porém não houveram resultados que influenciassem na sarcopenia (Dong et al., 2019).

Sabe-se que a combinação de exercícios aeróbicos e resistidos, também denominada como programa de exercícios combinados, possui efeitos benéficos de forma global alterando aspectos cardiopulmonares e musculares de forma mais completa e abrangente (Paim et al., 2021). Portanto, sabendo que a capacidade funcional e a força muscular desses pacientes podem estar prejudicadas, Yeh et al. (2020) buscaram analisar o efeito do ciclismo intradialítico no desempenho físico funcional com ganho de força e resistência muscular em pacientes com DRC avançada em hemodiálise. Foi realizado, neste caso, um programa de exercícios combinados através de um cicloergômetro que ofertava as duas modalidades de exercício por doze semanas, três vezes por semana, por trinta minutos, obtendo resultados significativos no aumento na distância percorrida do Teste de Caminhada de 6 minutos (TC6min), ou seja, uma melhora da capacidade submáxima e funcional de exercício e no Teste de Sentar e Levantar, que representa um aumento da força e resistência muscular de membros inferiores.

Huang et al. (2020) também investigaram os efeitos dos exercícios intradialíticos combinados através de um cicloergômetro que ofertava as duas modalidades de exercício na eficiência da hemodiálise, pressão arterial, capacidade de exercício e qualidade de vida. O programa consistia em quarenta minutos de exercício, realizados três vezes por semana, por vinte e quatro semanas. Por fim, encontrou-se um aumento na taxa Kt/V e na distância percorrida do TC6min, indicando uma melhor qualidade de diálise pós-exercício e aumento da capacidade submáxima de exercício. Houve também uma redução significativa da pressão arterial, sistólica e diastólica. Porém os resultados não mostraram alterações significativas nos índices de qualidade de vida.

Por ficarem várias horas diárias e muitos dias por semana submetido ao procedimento da hemodiálise, os pacientes com DRC costumam desenvolver elevados índices de inatividade física e referem um importante declínio em sua qualidade de sono, Cho et al. (2018), analisaram os efeitos do exercício intradialítico nestas variáveis. O programa consistiu na comparação dos exercícios aeróbicos através de bicicleta ergométrica, resistidos por meio de bandas elásticas e combinados, por doze semanas, três vezes por semana, durante quarenta minutos. Como resultados deste estudo, pôde-se observar melhora no equivalente metabólico (MET) ao realizar exercícios combinados, redução de sedentarismo em exercícios aeróbicos, e redução do índice de fragmentação do sono em todos os grupos de intervenção. Portanto, afirma-se que a aplicação de exercícios intradialíticos pode refletir positivamente na redução da inatividade física destes pacientes e na melhora da qualidade de sono.

Fortalecendo este resultado, Guimarães et al. (2011) encontraram que cerca de 80\% dos pacientes em hemodiálise apresentam qualidade de sono abaixo do satisfatório e que isto pode refletir significantemente na qualidade de vida. Com isso, Dias (2019) traz que o efeito vasodilador do exercício está diretamente relacionado a esta melhora do sono, associada aos demais benefícios.

Testal et al. (2018) investigaram em seu estudo que o programa de treinamento intradialítico poderia influenciar na dose diária de diálise e também reduzir a ocorrência de rebotes, fenômeno este onde a remoção dos eletrólitos do sangue é 
efetiva, mas este se mantém desproporcionalmente concentrados em outras regiões periféricas do corpo, o que seria prejudicial à eficácia da diálise realizada. Por isso, realizou-se um programa de exercícios combinados, resistidos isométricos e aeróbicos com cicloergômetro comparado à exercícios domiciliares, nestas condições, por doze semanas, três vezes por semana por 60 minutos. Porém não houveram resultados significativos que comprovasse a eficácia do exercício intradialítico na dose e no rebote pós-diálise.

Conforme a literatura, a realização de exercícios intradialíticos costumam refletir positivamente no rebote, sendo este um fator contribuinte à melhora da qualidade da diálise, em decorrência de alterações cardiovasculares e linfáticas promovidas pelo exercício. Porém, esta variável está diretamente relacionada a intensidade, modalidade e frequência do exercício, e também será refletido sobre ela o tempo de avaliação após a intervenção, sendo maior este tempo, maior a chance de aumento deste rebote (Pu et al., 2019).

Suhardjono et al. (2019) buscaram avaliar os efeitos dos exercícios intradialíticos aeróbicos versus somente exercícios combinados na capacidade física, fatores inflamatórios e no estado nutricional destes pacientes a fim de determinar qual o melhor programa de exercícios a ser ofertado à esta população. Foi realizado um programa baseado em exercícios com caneleiras e cicloergômetro, duas vezes na semana por 20 minutos em um período de doze semanas. Observou-se que em ambos os grupos de exercício houve um aumento na força muscular de membros inferiores e na pontuação no componente físico do instrumento Kidney Disease and Quality-of-Life Short-Form (KDQOL-SFTM) que avalia qualidade de vida. Sendo assim, não se pode afirmar que exercícios combinados foram superiores ao exercício aeróbico nestes aspectos.

Outro desfecho clínico altamente encontrado é a hipotensão induzida pela hemodiálise. Diante disto, Álvares et al. (2017) compararam os efeitos da reabilitação intradialítica por exercícios aeróbicos versus terapia por compressão pneumática na redução da hipotensão intradialítica. O estudo foi aplicado por três semanas, três vezes na semana com sessenta minutos de duração. Um grupo realizou exercício aeróbico por meio de um cicloergômetro e outro recebeu compressão por meio de um sistema compressor de membros inferiores. Observou-se efeito positivo, concluindo que a compressão pneumática na primeira hora de hemodiálise contribuiu para redução da hipotensão, porém não houveram efeitos na remoção dos principais fluidos avaliados. Nem o exercício aeróbico e nem a compressão pneumática aumentaram a remoção de fosfato.

Diferente dos estudos anteriormente citados, Ortí et al. (2018) propuseram uma comparação entre os efeitos do exercício de realidade virtual versus exercícios convencionais aeróbicos e resistidos na função física dos pacientes em hemodiálise. Foram realizados trinta minutos de exercícios, três vezes na semana por quatro semanas. Como resultados, foi possível observar um aumento da função física em ambos os grupos. Podendo ser o exercício com uso de realidade virtual uma oportunidade de variabilidade no programa de exercícios intradialíticos.

Estudos também buscaram analisar o efeito de diferentes programas de eletroestimulação neuromuscular em alternados desfechos relacionados aos pacientes com DRC em hemodiálise. A eletroestimulação é usada em programas de reabilitação a fim de promover aumento do trofismo, fortalecimento e melhora do desempenho muscular através de correntes elétricas que produzem contração muscular (Freitas, 2012).

Bruggerman et al. (2017) buscaram analisar os efeitos da eletroestimulação neuromuscular na função física e em marcadores inflamatórios dos pacientes com DRC, comparando ainda a eficiência de um programa de alta intensidade e frequência versus baixa intensidade e frequência. Foi realizado um programa de quatro semanas, onde os pacientes recebiam o tratamento por sessenta minutos, três vezes na semana. Ambos os grupos obtiveram um aumento da distância percorrida no TC6min, mas apenas o grupo que recebeu altas intensidades e frequências obtiveram um aumento da força muscular periférica, redução dos níveis de Interleucina-10 e fatores pró-inflamatórios. Em contrapartida, apenas o grupo que recebeu baixas intensidades e frequências aumentou os níveis dos fatores de crescimento semelhante à insulina tipo 1 (IGF-1). Portanto pode- 
se concluir conforme o estudo que, os pacientes com DRC podem ter sua função física beneficiada pela eletroestimulação em geral, porém seus marcadores inflamatórios podem ter resultados específicos conforme o programa de reabilitação aplicado.

Complementando o estudo anterior, Schardong et al. (2017) avaliaram a ação da eletroestimulação neuromuscular na força e arquitetura muscular, capacidade funcional e função endotelial de pacientes com DRC. Realizaram oito semanas de intervenção, iniciando com 20 minutos chegando a 34 minutos, incrementando 2 minutos a cada semana, três vezes por semana. O presente estudo conseguiu resultados positivos no aumento da força de membros inferiores e na proteção da atrofia muscular nestes pacientes. Porém não foram evidenciadas mudanças na capacidade funcional e na função endotelial.

Conforme a literatura, já se sabe sobre a consolidação dos resultados da eletroestimulação em propriedades neuromusculares, porém quando fala-se sobre a função endotelial, Karavidas et al. (2013) nos trazem que uma melhora desta é dependente da intensidade, volume e tempo de tratamento, sendo encontrados efeitos positivos quando aplicadas por períodos maiores de intervenção, podendo esta ter sido uma limitação do estudo de Schardong et al.

Ainda, quanto aos estudos que utilizaram eletroestimulação neuromuscular, Susuki et al. (2018) implementaram um programa que aplicou a intervenção por oito semanas, três vezes na semana por cento e vinte minutos, a fim de avaliar seus efeitos de forma intradialítica. Foi evidenciada um aumento de força e trofismo do músculo quadríceps e também aumento em todos os componentes do instrumento de qualidade de vida, SF-8, porém estes não foram estatisticamente significativos, não podendo seu resultado ser considerado.

Além das avaliações e busca por resultados musculares nos pacientes com DRC, Yang et al. (2018) buscaram utilizar a aplicação de estimulação elétrica nervosa transcutânea (TENS) para reduzir a sensação de boca seca e aumentar a taxa de fluxo salivar nos pacientes que realizam hemodiálise, já que esta é uma queixa frequente destes pacientes e um incômodo para grande parte deles. O TENS foi aplicado por vinte minutos, três vezes na semana, por três semanas de intervenção, porém os pacientes foram reavaliados com duas, três, quatro e cinco semanas. No final de cinco semanas, foi possível constatar um aumento estável da taxa de fluxo salivar e uma redução na intensidade da sensação de boca seca nestes pacientes.

Outros estudos tentaram buscar efeitos da aplicação da fotobiomodulação intradialítica, que consiste na aplicação de uma luz aos sistemas que irá induzir a formação de um processo fotoquímico, que irá estimular as mitocôndrias e o processo de produção de energia. Isto irá estimular a produção de ATP o que pode aumentar o metabolismo celular (Bacelete \& Gama, 2021). Por isso, Macagnan et al. (2018) e Schardong et al. (2020) buscaram compreender diferentes desfechos aos pacientes com DRC em hemodiálise após aplicação de fotobiomodulação. Macagnan et al. (2018) buscaram compreender os efeitos de três diferentes doses de laser na força de preensão palmar destes pacientes. O tratamento foi realizado por quatro semanas, com intervalo de uma semana entre as aplicações que eram realizadas uma vez na semana, o tempo de aplicação não foi citado. Como resultado foi possível constatar um aumento significativo da força durante a aplicação de 60 e 90 Joules (J) por braço enquanto nenhuma alteração foi observada na aplicação de $120 \mathrm{~J} /$ braço. Portanto, compreende-se que a terapia por fotobiomodulação pode ser uma alternativa para aumento da força nos pacientes com DRC, porém não é necessária a aplicação de doses mais elevadas para esta finalidade.

Já Schardong et al. (2020) aplicaram um programa oito semanas de laserterapia, três vezes por semana a fim de investigar os efeitos da fotobiomodulação na capacidade funcional e secundariamente na força, espessura muscular e ecogenicidade, percepção da dor, fadiga e qualidade de vida dos pacientes com DRC. Pôde-se observar um aumento da capacidade funcional e na força muscular dos membros inferiores dos pacientes que receberam a intervenção. Porém para os outros desfechos não foram encontrados resultados significativos.

Fortalecendo os resultados encontrados, sabe-se que a laserterapia não representa uma indicação primária para desfechos que busquem alterações de mudanças na estrutura muscular. E ainda, métodos avaliativos precisos devem ser 
aplicados quando avaliadas dor e fadiga, a fim de objetivar os resultados encontrados. Quando o objetivo for dor, a laserterapia deve ser aplicada em regiões maiores a fim de alterar este limiar, podendo este fator contribuir ao resultado negativo no estudo de Schardong et al. (Vanin et al., 2016).

Roxo et al. (2016) buscaram compreender os efeitos da eletroestimulação neuromuscular na função pulmonar e na capacidade funcional dos pacientes em hemodiálise de manutenção. A terapia consistiu em trinta minutos de eletroestimulação em quadríceps, realizados três vezes por semana, em um programa de oito semanas. Como resultados obteve-se um aumento da pressão inspiratória máxima, aumento do teste de uma repetição máxima (1RM) e na distância percorrida no TC6min. Houve também uma redução da pressão arterial sistólica e da frequência respiratória. Com isso, pode-se afirmar que a eletroestimulação contribuiu positivamente para o desempenho físico e pulmonar dos pacientes com DRC.

Sendo assim, Figueiredo et al. (2018) avaliaram os efeitos combinados e isolados do treinamento muscular inspiratório com Threshold, Power-Breathe e aeróbico nos parâmetros respiratórios e funcionais, biomarcadores inflamatórios, estado redox, que representa um fenômeno químico produzindo energia a partir de uma oxidação celular, e também da qualidade de vida relacionada à saúde em pacientes em hemodiálise. O programa foi realizado em oito semanas com acompanhamento em dezesseis semanas, três vezes na semana realizado por quarenta minutos de exercício aeróbico e quinze inspirações para o treinamento muscular inspiratório. Ambos os grupos tiveram melhoras nos parâmetros funcionais (capacidade funcional, pressão inspiratória máxima e força de membros inferiores) e nos biomarcadores inflamatórios, considerando que o treinamento muscular inspiratório e aeróbico isoladamente e de forma combinada apresentaram os mesmos resultados.

Semelhante ao estudo supracitado, Yuenyongchaiwat et al. (2020) utilizaram o treinamento muscular inspiratório para melhorar aptidão respiratória, qualidade de vida e redução da falta de ar. Realizou-se treinamento com Threshold por tempo de oito semanas, quinze inspirações máximas, três séries com sessenta segundos de repouso, três vezes por semana. Foi possível observar após intervenção um aumento da força muscular inspiratória e da capacidade vital forçada. Não houveram mudanças significativas em parâmetros de qualidade de vida.

Quanto aos achados negativos relacionados à qualidade de vida, Carvalho et al. (2020) correlaciona esta alteração à intensidade do exercício, sendo superiores em exercícios em intensidades moderadas a altas. E também são citados exercícios domiciliares como uma alternativa mais eficiente nestes pacientes.

Devemos considerar que toda intervenção pode ocasionar efeitos adversos, incluindo também a fisioterapia intradialítica. Neste caso, podem ocorrer deslocamentos dos acessos vasculares e até mesmo uma desestabilização hemodinâmica destes pacientes. Mas sabe-se que os efeitos de uma imobilidade podem ser altamente deletérios, se sobressaindo aos riscos das sessões intradialíticas. Mas reforça-se a importância de uma prescrição de exercícios segura e viável, pautada por uma avaliação fisioterapêutica individualizada (Fernandes \& Gardenghi, 2019).

Apesar dos exercícios intradialíticos estarem bem elucidados na literatura, a implementação destes programas ainda são um desafio dentro dos grandes centros, por barreiras levantadas pelos pacientes e também por falta de recursos. Porém o presente estudo possui importante relevância ao cenário clínico do tratamento destes pacientes, comprovando que programas de reabilitação intradialíticos podem proporcionar aos pacientes renais crônicos um tratamento de melhor qualidade, atendendo aspectos fisiológicos, mas também características físico-funcionais que podem refletir positivamente em sua saúde e bem-estar.

Como limitações, podem ser citadas as variabilidades de intervenções que podem ter influenciado na comparação de resultados, por isso acredita-se que novas análises, com maior quantidade de estudos inclusos possam ofertar uma melhor qualidade metodológica e ainda, que estes possam especificar as abordagens de forma isolada, a fim de contribuir com variáveis específicas de prescrição de exercício nesta população. 


\section{Considerações Finais}

Conforme a análise realizada neste trabalho, os estudos podem ser considerados de moderada a boa qualidade metodológica pela Escala PEDro quanto à aplicação de intervenções intradialítica. Porém, itens relacionados ao cegamento da amostra, dos avaliadores e dos aplicadores das intervenções foram os pontos mais deficientes conforme a análise. Portanto sugere-se que estes sejam realizados em estudos futuros para uma melhor qualidade metodológica em futuras publicações.

As principais intervenções aplicadas foram exercícios combinados (aeróbicos e resistidos), exercícios aeróbicos, exercícios resistidos e eletrotermofototerapia. Estes refletiram positivamente em aspectos físicos como força muscular, capacidade funcional e capacidade de realização de exercício, função física, redução da inatividade física, modulação de fatores de risco cardiovasculares e melhora da qualidade do sono. E ainda, com a aplicação de exercício intradialítico foi possível a modulação e melhora de aspectos relacionados aos biomarcadores inflamatórios e qualidade da diálise destes pacientes.

Alguns desfechos secundários também foram encontrados como a redução da hipotensão induzida pela hemodiálise através da compressão pneumática. Além disso, observou-se o aumento da taxa de fluxo salivar e redução da sensação de boca seca através de eletroterapia intradialítica. Estes podem contribuir positivamente para as queixas dos pacientes.

Portanto, sugere-se que um programa de exercícios intradialíticos possa contribuir positivamente para os pacientes com Doença Renal Crônica, selecionando a modalidade de exercício conforme a necessidade do paciente. Sendo o programa com maiores resultados aqueles realizados três vezes na semana, por no mínimo doze semanas.

Muitos estudos buscaram compreender os efeitos de suas intervenções nos aspectos da qualidade de vida, esta altamente reduzida nos pacientes que realizam hemodiálise de manutenção. Porém, poucos obtiveram resultados significativos nestes indicadores. Portanto, sugere-se que em estudos futuros se busque resultados de intervenções intradialíticas com o desfecho primário voltado para melhorias da qualidade de vida e estado funcional, com uma reabilitação de pacientes com DRC ampla, compreendendo aspectos físicos, fisiológicos e psicossociais.

\section{Referências}

Álvares, V. R. C., Ramos, C. D., Pereira, B. J., Pinto, A. L., Moysés, R. M. A., Gualano, B., \& Elias, R. M. (2017). Pneumatic Compression, But Not Exercise, Can Avoid Intradialytic Hypotension: A Randomized Trial. American Journal of Nephrology, 45(5), 409-416. https://doi.org/10.1159/000471513

Bacelete, V. S. B., \& Gama, A. C. C. (2021). Therapeutic effects of photobiomodulation in the speech-language-hearing clinic: An integrative literature review. Revista CEFAC, 23(1). https://doi.org/10.1590/1982-0216/20212319120

Bastos, M. G., Bregman, R., \& Kirsztajn, G. M. (2010). Doença renal crônica: Frequente e grave, mas também prevenível e tratável. Revista da Associação Médica Brasileira, 56(2), 248-253. https://doi.org/10.1590/s0104-42302010000200028

Brasil. Ministério da Saúde. (2014). Diretrizes clínicas para o cuidado ao paciente com doença renal crônica-DRC no Sistema Único de Saúde. https://portalarquivos2.saude.gov.br/images/pdf/2014/marco/24/diretriz-cl--nica-drc-versao-final.pdf.

Breitsameter, G., Figueiredo, A. E., \& Kochhann, D. S. (2012). Cálculo de Kt/V em hemodiálise: Comparação entre fórmulas. Jornal Brasileiro de Nefrologia, 34(1), 22-26. https://doi.org/10.1590/s0101-28002012000100004

Brüggemann, A. K., Mello, C. L., Dal Pont, T., Hizume Kunzler, D., Martins, D. F., Bobinski, F., Pereira Yamaguti, W., \& Paulin, E. (2017). Effects of Neuromuscular Electrical Stimulation During Hemodialysis on Peripheral Muscle Strength and Exercise Capacity. Archives of Physical Medicine and Rehabilitation, 98(5), 822-831.e1. https://doi.org/10.1016/j.apmr.2016.12.009

Carvalho, A. R., Sousa, I. M. d., Silva, D. H. d., Silva, A. R. d., Adad, R. B. S. d. F., \& Silva, V. O. d. (2020). Os efeitos do exercício físico em pacientes submetidos à hemodiálise: uma revisão sistemática. Revista Pesquisa em Fisioterapia, 10(2), 309. https://doi.org/10.17267/2238-2704rpf.v10i2.2638

Cho, J.-H., Lee, J.-Y., Lee, S., Park, H., Choi, S.-W., \& Kim, J. C. (2018). Effect of intradialytic exercise on daily physical activity and sleep quality in maintenance hemodialysis patients. International Urology and Nephrology, 50(4), 745-754. https://doi.org/10.1007/s11255-018-1796-y

Costa, J. A., \& Damasceno, M. J. F. (2018). Benefícios do treinamento resistido em pacientes com doença renal crônica em tratamento de hemodiálise. Health Research Journal, 1(3), 18-32.

Dias, H. S. (2019). O efeito do exercício físico em pacientes submetidos ao tratamento de hemodiálise. Revista Brasileira de Fisiologia do Exercício, 18(1), 58. https://doi.org/10.33233/rbfe.v18i1.2880 
Doenças renais: Causas, sintomas, diagnóstico, tratamento e prevenção. (2019, 14 de março). Ministério da Saúde. https://antigo.saude.gov.br/saude-de-az/doencas-renais.

Dong, Z.-J., Zhang, H.-L., \& Yin, L.-X. (2019). Effects of intradialytic resistance exercise on systemic inflammation in maintenance hemodialysis patients with sarcopenia: a randomized controlled trial. International Urology and Nephrology, 51(8), 1415-1424. https://doi.org/10.1007/s11255-019-02200-7

Escala PEDro - PEDro. (1999). PEDro. https://pedro.org.au/portuguese/resources/pedro-scale/.

Fernandes, P. N. R., \& Gardenghi, G. (2019). Fisioterapia intradialítica nas unidades de terapia intensiva, uma revisão sobre as barreiras, segurança e viabilidade. 9(1), 44-54. https://rescceafi.com.br/vol9/n1/artigo5_pags_44a54.pdf

Figueiredo, P. H. S., Lima, M. M. O., Costa, H. S., Martins, J. B., Flecha, O. D., Gonçalves, P. F., Alves, F. L., Rodrigues, V. G. B., Maciel, E. H. B., Mendonça, V. A., Lacerda, A. C. R., Vieira, É. L. M., Teixeira, A. L., de Paula, F., \& Balthazar, C. H. (2018). Effects of the inspiratory muscle training and aerobic training on respiratory and functional parameters, inflammatory biomarkers, redox status and quality of life in hemodialysis patients: A randomized clinical trial. Plos One, 13(7), Artigo e0200727. https://doi.org/10.1371/journal.pone.0200727

Freitas, T. P. (2016). Efeitos da eletroestimulação neuromuscular e exercício resistido sobre a atividade elétrica e força do bíceps braquial. Fisioterapia Brasil, 13(3). https://doi.org/10.33233/fb.v13i3.539

Guimarães, C. K. D., Alves, D. A. G., \& Guimarães, L. H. d. C. T. (2011). Avaliação da qualidade e quantidade do sono em pacientes renais crônicos submetidos à hemodiálise. Revista Neurociências, 19(4), 609-613. https://doi.org/10.34024/rnc.2011.v19.8322

Huang, M., Lv, A., Wang, J., Zhang, B., Xu, N., Zhai, Z., Gao, J., Wang, Y., Li, T., \& Ni, C. (2020). The effect of intradialytic combined exercise on hemodialysis efficiency in end-stage renal disease patients: A randomized-controlled trial. International Urology and Nephrology, 52(5), 969-976. https://doi.org/10.1007/s11255-020-02459-1

Jesus, N. M., Souza, G. F. D., Mendes-Rodrigues, C., Almeida, O. P. D., Rodrigues, D. D. M., \& Cunha, C. M. (2019). Qualidade de vida de indivíduos com doença renal crônica em tratamento dialítico. Brazilian Journal of Nephrology, 41, 364-374.

Júnior, C. M. A. L., Lima, W. P., Souza, L. M. V., Souza, C. M. S., Dos Santos, J. R., Costa, F. B., Silva, A. L. d. S., De Jesus, E. V., Carvalho, P. E., \& Dantas, E. H. M. (2021). O treinamento físico como estratégia de intervenção em pessoas com Insuficiência Renal Crônica. Revista Eletrônica Acervo Saúde, 13(2), Artigo e6140. https://doi.org/10.25248/reas.e6140.2021

Junior, J. E. R. (2004). Doença renal crônica: definição, epidemiologia e classificação. J. Bras. Nefrol., 26 (3 suppl. 1), 1-3.

Lima, A. P. F., Rocha, B. S., Menezes, I. H. C. F., \& Pereira, E. R. S. (2021). Refletindo sobre a Educação Permanente em Saúde: potencialidades e limitações na terapia renal substitutiva. Interface-Comunicação, Saúde, Educação, 25. https://doi.org/10.1590/interface.200494

Macagnan, F. E., Baroni, B. M., Cristofoli, É. Z., Godoy, M., Schardong, J., \& Plentz, R. D. M. (2018). Acute effect of photobiomodulation therapy on handgrip strength of chronic kidney disease patients during hemodialysis. Lasers in Medical Science, 34(4), 835-840. https://doi.org/10.1007/s10103-0182593-7

Neves, P. D. M. D. M., Sesso, R. D. C. C., Thomé, F. S., Lugon, J. R., \& Nasicmento, M. M. (2020). Censo Brasileiro de Diálise: análise de dados da década 2009-2018. Brazilian Journal of Nephrology, 42, 191-200.

Orcy, R. B., Dias, P. S., Seus, T. L., Barcellos, F. C., \& Bohlke, M. (2012). Combined Resistance and Aerobic Exercise is Better than Resistance Training Alone to Improve Functional Performance of Haemodialysis Patients - Results of a Randomized Controlled Trial. Physiotherapy Research International, 17(4), 235-243. https://doi.org/10.1002/pri.1526

Ortí, E. S., Domínguez, B. P., Villar, L. O. d., Oliva, E. M., Gramage, J. M., Maset, R. G., \& Gómez, J. A. G. (2018). Virtual reality exercise intradialysis to improve physical function: A feasibility randomized trial. Scandinavian Journal of Medicine \& Science in Sports, 29(1), 89-94. https://doi.org/10.1111/sms.13304

Paim, F., Somavilla, A. B., Mayer, F. D. A., Kupske, J. W., Moreira, P. R., \& Krug, R. D. R. (2021, July). MAPEAMENTO DOS PROGRAMAS DE EXERCÍCIOS FÍSICOS INTRADIALÍTICOS NO BRASIL. In Congresso Internacional em Saúde (No. 8).

Powers, S. K., \& Howley, E. T. (2014). Fisiologia do Exercício: Teoria e Aplicação ao Condicionamento e ao Desempenho (8a ed.). Manole.

PRISMA. (2020). PRISMA. http://www.prisma-statement.org/

Pu, J., Jiang, Z., Wu, W., Li, L., Zhang, L., Li, Y., Liu, Q., \& Ou, S. (2019). Efficacy and safety of intradialytic exercise in haemodialysis patients: a systematic review and meta-analysis. BMJ Open, 9(1), Artigo e020633. https://doi.org/10.1136/bmjopen-2017-020633

Ramos, E. C. C., Santos, I. D. S. D., Zanini, R. D. V., \& Ramos, J. M. G. (2015). Qualidade de vida de pacientes renais crônicos em diálise peritoneal e hemodiálise. Brazilian Journal of Nephrology, 37, 297-305.

Rosa, C. S. d. C., Nishimoto, D. Y., Souza, G. D. e., Ramirez, A. P., Carletti, C. O., Daibem, C. G. L., Sakkas, G. K., \& Monteiro, H. L. (2018). Effect of continuous progressive resistance training during hemodialysis on body composition, physical function and quality of life in end-stage renal disease patients: A randomized controlled trial. Clinical Rehabilitation, 32(7), 899-908. https://doi.org/10.1177/0269215518760696

Roxo, R. S., Xavier, V. B., Miorin, L. A., Magalhães, A. O., Sens, Y. A. d. S., \& Alves, V. L. d. S. (2016). Impact of neuromuscular electrical stimulation on functional capacity of patients with chronic kidney disease on hemodialysis. Jornal Brasileiro de Nefrologia, 38(3). https://doi.org/10.5935/01012800.20160052

Santos, B. P., Oliveira, V. A., Soares, M. C., \& Schwartz, E. (2017). Doença renal crônica: relação dos pacientes com a hemodiálise. ABCS Health Sciences, $42(1)$. 
Schardong, J., Dipp, T., Bozzeto, C. B., da Silva, M. G., Baldissera, G. L., Ribeiro, R. d. C., Valdemarca, B. P., do Pinho, A. S., Sbruzzi, G., \& Plentz, R. D. M. (2017). Effects of intradialytic neuromuscular electrical stimulation on strength and muscle architecture in patients with chronic kidney failure: Randomized clinical trial. Artificial Organs, 41(11), 1049-1058. https://doi.org/10.1111/aor.12886

Schardong, J., Falster, M., Sisto, I. R., Barbosa, A. P. O., Normann, T. C., de Souza, K. S., Jaroceski, G., Bozzetto, C. B., Baroni, B. M., \& Plentz, R. D. M. (2020). Photobiomodulation therapy increases functional capacity of patients with chronic kidney failure: Randomized controlled trial. Lasers in Medical Science, 36(1), 119-129. https://doi.org/10.1007/s10103-020-03020-3

Shiwa, S. R., Costa, L. O. P., Moser, A. D. D. L., Aguiar, I. D. C., \& Oliveira, L. V. F. D. (2011). PEDro: a base de dados de evidências em fisioterapia. Fisioterapia em Movimento, 24, 523-533.

Silva, V. R. O. e., Belik, F. S., Hueb, J. C., Gonçalves, R. d. S., Caramori, J. C. T., Vogt, B. P., Barretti, P., Bazan, S. G. Z., Stefano, G. M. M. F., Martin, L. C., \& Franco, R. J. d. S. (2019). Aerobic Exercise Training and Nontraditional Cardiovascular Risk Factors in Hemodialysis Patients: Results from a Prospective Randomized Trial. Cardiorenal Medicine, 9(6), 391-399. https://doi.org/10.1159/000501589

Sociedade Brasileira de Nefrologia (SBN). Diálise peritoneal (2021, 3 de março). https://www.sbn.org.br/orientacoes-e-tratamentos/tratamentos/dialiseperitoneal/.

Sociedade Brasileira de Nefrologia (SBN). Doença renal crônica: Diagnóstico e prevenção. (2021, 3 de março). https://www.sbn.org.br/noticias/single/news/doenca-renal-cronica-diagnostico-e-prevencao/

Sociedade Brasileira de Nefrologia (SBN). Hemodiálise - SBN. (2021, 3 de março). https://www.sbn.org.br/orientacoes-etratamentos/tratamentos/hemodialise/

Soto, M. G. O., Ortiz, R. V., Alvarenga, J. C. L., \& Cuevas, M. d. 1. Á. E. (2016). Effect of Resistance Exercises on the Indicators of Muscle Reserves and Handgrip Strength in Adult Patients on Hemodialysis. Journal of Renal Nutrition, 26(1), 53-60. https://doi.org/10.1053/j.jrn.2015.06.006

Souza, R. L. (2018). Aplicação de um programa de fisioterapia em pacientes com insuficiência renal crônica. Revista Experiências e Evidências em Fisioterapia e Saúde-ISSN 2595-7872, 1(1). http://www.cescage.com.br/revistas/index.php/Exper_Evid_Fisioterapia/article/view/165.

Suhardjono, V. U., Tedjasukmana, D., \& Setiati, S. (2019). The effect of intradialytic exercise twice a week on the physical capacity, inflammation, and nutritional status of dialysis patients: A randomized controlled trial. Hemodialysis International, 23(4), 486-493. https://doi.org/10.1111/hdi.12764

Suzuki, T., Ikeda, M., Minami, M., Matayoshi, Y., Nakao, M., Nakamura, T., \& Abo, M. (2018). Beneficial effect of intradialytic electrical muscle stimulation in hemodialysis patients: A randomized controlled trial. Artificial Organs, 42(9), 899-910. https://doi.org/10.1111/aor.13161

Testal, A. G., Maset, R. G., Marín, D. H., Domínguez, B. P., Maicas, P. R., Salvador, I. S. R., Oliva, E. M., Aracil, J. M., Chiva, M. M., Blanco, O. D. P., Díaz, P. O., Najera, J. E. F., Juan, E. T. D., Carrera, C. B., \& Ortí, E. S. (2018). Influence of physical exercise on the dialytic adequacy parameters of patients on hemodialysis. Therapeutic Apheresis and Dialysis, 23(2), 160-166. https://doi.org/10.1111/1744-9987.12762

Vanin, A. A., Marchi, T. D., Tomazoni, S. S., Tairova, O., Casalechi, H. L., Carvalho, P. d. T. C. d., Bjordal, J. M., \& Junior, E. C. L. (2016). Pre-Exercise Infrared Low-Level Laser Therapy $(810 \mathrm{~nm})$ in Skeletal Muscle Performance and Postexercise Recovery in Humans, What Is the Optimal Dose? A Randomized, Double-Blind, Placebo-Controlled Clinical Trial. Photomedicine and Laser Surgery, 34(10), 473-482. https://doi.org/10.1089/pho.2015.3992

Yang, L., Chen, H., Su, Y., \& Chin, C. (2018). The effect of transcutaneous electrical nerve stimulation on increasing salivary flow rate in hemodialysis patients. Oral Diseases, 25(1), 133-141. https://doi.org/10.1111/odi.12948

Yeh, M.-L., Wang, M.-H., Hsu, C.-C., \& Liu, Y.-M. (2020). Twelve-week intradialytic cycling exercise improves physical functional performance with gain in muscle strength and endurance: A randomized controlled trial. Clinical Rehabilitation, 34(7), 916-926. https://doi.org/10.1177/0269215520921923

Young, H. M. L., March, D. S., Highton, P. J., Graham-Brown, M. P. M., Churchward, D. C., Grantham, C., Goodliffe, S., Jones, W., Cheung, M.-M., Greenwood, S. A., Eborall, H. C., Conroy, S., Singh, S. J., Smith, A. C., \& Burton, J. O. (2020). Exercise for people living with frailty and receiving haemodialysis: A mixed-methods randomised controlled feasibility study. BMJ Open, 10(11), Artigo e041227. https://doi.org/10.1136/bmjopen-2020-041227

Yuenyongchaiwat, K., Namdang, P., Vasinsarunkul, P., Phongsukree, P., Chaturattanachaiyaporn, K., Pairojkittrakul, S., \& Traitanon, O. (2020). Effectiveness of inspiratory muscle training on respiratory fitness and breathlessness in chronic renal failure: A randomized control trial. Physiotherapy Research International. https://doi.org/10.1002/pri.1879.

Waclawovsky, G., Pedralli, M. L., Eibel, B., Schaun, M. I., \& Lehnen, A. M. (2021). Efeitos de Diferentes Tipos de Treinamento Físico na Função Endotelial em Pré-Hipertensos e Hipertensos: Uma Revisão Sistemática. Arquivos Brasileiros de Cardiologia, 116(5), $938-947$. 